\title{
Efficient Zero-Knowledge Proofs of Knowledge of Double Discrete Logarithm
}

\author{
Bin Lian ${ }^{1,2}$, Gongliang Chen ${ }^{1}$ and Jianhua $\mathrm{Li}^{1}$ \\ ${ }^{1}$ School of Information Security Engineering, Shanghai Jiao Tong University, \\ Shanghai, China \\ ${ }^{2}$ Ningbo Institute of Technology, Zhejiang University, Ningbo, China \\ lianbin_a@163.com,chengl@sjtu.edu.cn,Lijh888@sjtu.edu.cn
}

\begin{abstract}
Zero-knowledge proof protocol is a basic cryptographic technique. And zero-knowledge proof of double discrete logarithm has some particular properties, so it has been widely applied in many security systems. But the efficient problem of zero-knowledge proof of double discrete logarithm has not been solved to this day, since there are some special difficulties in computing this kind of knowledge proof. Hence, the time complexity and the space complexity of existing schemes are all $O(k)$, where $k$ is a security parameter. After redesigning the basic construction of knowledge proof, we provide a new zero-knowledge proof of double discrete logarithm, which is the first scheme with $O(1)$ time complexity and $O(1)$ space complexity. If introducing an off-line TTP (trusted third party), we can provide two additional zeroknowledge proof schemes of double discrete logarithm, one is even more efficient than the first one, the other one solves another open problem, which is how to efficiently prove the equality of double discrete logarithms in zero-knowledge way, and the existing techniques cannot solve this problem. We also provide the detailed security proofs of our designs and efficiency analysis, comparing with the existing schemes. The significant improvement in efficiency of this basic cryptographic technique is also helpful for many security systems.
\end{abstract}

Keywords: zero-knowledge proof of double discrete logarithm, signature based on proofs of knowledge, constant complexity, proof of equality of double discrete logarithms

\section{Introduction}

Zero-knowledge proofs are defined as the proofs which convey no additional knowledge other than the correctness of the proposition in question.

An interactive proof $[1,2]$ of a proposition is an interactive protocol between two entities, a prover and a verifier. After performing the protocol, the verifier is convinced of the correctness of the proposition. Interactive proofs have one remarkable advantage compared to conventional proofs, which is that, there exist protocols for proving a proposition such that the verifier does not receive any information apart from the correctness of the proposition. So the verifier is not able to prove the proposition to other people. Furthermore, the information which the verifier obtains seems completely random to him and the verifier is always convinced of the correctness of the proposition after performing the protocol if he is honest. This property is called honest-verifier zero-knowledge.

The original definition of zero-knowledge was provided in [3] and their applicability to any language L in NP was demonstrated in [4]. [5] Showed that all statements in NP have a zero-knowledge proof-system, where the prover is computationally unbounded, while the verifier is a probabilistic polynomial-time machine. Then [6-8] modified the original model 
such that the prover is a (probabilistic) polynomial-time machine, where either the statement is in $\mathbf{P}$ (i.e., the class of problems which can be decided in polynomial time), or the prover must 'know' a witness, i.e., store the witness or compute it in polynomial time from other stored information. And the protocols in this model are usually called proofs of knowledge.

As a basic module, the zero-knowledge proofs of knowledge have been widely applied in many cryptographic systems [9-26]. There are various zero-knowledge proofs of different knowledge, such as knowledge of discrete logarithm, knowledge of representation of an element, knowledge of equality of discrete logarithms, knowledge of polynomial relations of discrete logarithms and so on [10,13,27]. And more security proofs and extended modes of zero-knowledge proofs are in [28]. The general method on designing zero-knowledge proofs can be seen in [29].

One kind of zero-knowledge proofs is the zero-knowledge proof of knowledge of double discrete logarithm, where the double discrete logarithm of $y \in \mathrm{G}$ to bases $g$ and $u$ is the integer $x \in \mathrm{Z}_{\mathrm{q}}$ satisfying $y=g^{u^{x}}(\bmod n)$ if this $x$ exists. The zero-knowledge proof of knowledge of double discrete logarithm has also been used in many kinds of security systems, such as Ecash system, signature scheme supporting concurrent issue, group signature scheme, publicly verifiable secret sharing scheme, public key algorithm, designated verifier signature scheme and so on $[10,13,19,27,30-39]$. Since it acts as the fundamental part of these security systems, the zero-knowledge proof of knowledge of double discrete logarithm has attracted researchers' attentions. [40] analyzed the exact complexity of double discrete logarithm problem and [41] studied the interpolation problem of double discrete logarithm. And their results supported the assumption of hardness of the double discrete logarithm.

However, the efficiency problem of zero-knowledge proof of knowledge of double discrete logarithm has not been solved yet, though it has been widely applied in so many systems. In fact, the realization mode of zero-knowledge proof of double discrete logarithm has not been improved in efficiency since it was been proposed. The time complexity and the space complexity of existing zero-knowledge proof of double discrete logarithm are all $O(k)$ where $k$ is a system parameter, and in their system $[10,13,19,27,30-32,35,36,39]$, the probability of forging the knowledge proof is $1 / 2^{k}$. And since $k>80$ in applications, the existing proofs of double discrete logarithm are all inefficient comparing with other zero-knowledge proofs. Being different from the common exponential computation, the double exponent is hard to combine with the challenge information from the verifier when constructing the knowledge proof because of the high computation complex. So the existing idea of knowledge proof of double discrete logarithm is that the challenge information decides the response from the prover bit by bit, and it results in the poor efficiency. Therefore, it is hard to improve the efficiency of the existing schemes based on this idea.

We solve the efficiency problem of knowledge proof of double discrete logarithm in an original way. For solving the problem, we design a new zero-knowledge proof of discrete logarithm, which acts as the basic construction of our zero-knowledge proof of double discrete logarithm. And because the new knowledge proof changes the standard form of knowledge proof, we provide the detailed security proofs of the new basic construction. Based on the basic construction, we design the first efficient zero-knowledge proof of knowledge of double discrete logarithm. Because of the change of basic construction, we improve the related efficiency by one order of magnitude. The system complexity is improved from existing $O(k)$ to $O(1)$. If introducing an off-line TTP (trusted third party), we also design another two zero-knowledge proofs of double discrete logarithms. One achieves even better efficiency, and the other one achieves knowledge proof of equality of double discrete logarithms, which is also the first proof of equality of double discrete logarithms. And the proof of equality of double discrete logarithms has not been studied before, because 
the existing method cannot provide a feasible way to achieve it. [27, 36] considered one similar case, but [27, 36] also cannot provide a feasible (efficient and practical) zeroknowledge proof of equality of double discrete logarithms, because their idea is based on traditional inefficient method, i.e., the cut-and-choose method, so [27] also thinks that it is an open problem.

Using the techniques of [42], the honest-verifier zero-knowledge proof can be turned into a signature scheme by replacing the verifier by a hash function, i.e., the challenge is set to the hash value of the commitment (and of the message to be signed). So as the existing scheme $[10,13,19,27,30-32,35,39]$ did, we also provide the noninteractive version of our three protocols of zero-knowledge proofs, i.e., the signature based on proofs of knowledge (SPK).

The remainder of this paper is organized as follows. The section 2 gives preliminaries of our design, and in particular, the existing knowledge proof of double discrete logarithm is presented. In the section 3, we provide the new zero-knowledge proofs of knowledge of double discrete logarithms and the SPK of the proofs, and we also provide the security proofs of these designs. In the section 4, we analyze system efficiency. Finally, the paper concludes in the section 5 .

\section{Preliminaries}

\subsection{Cryptographic Assumptions}

Problem 1 (Strong RSA Problem). For given (n, z), finding $u \in G$ and $e \in Z_{>1}$ satisfying $z \equiv u^{e}$ $(\bmod n)$, where $n=p q$ is an RSA-like modulus and $G$ is a cyclic subgroup of $Z_{n}{ }_{n}$ of order $\# G$, $\left.{ }_{\log }(\# G)\right\rceil=l_{G}$.

Assumption 1 (S-RSA Assumption) [43, 44]. A probabilistic polynomial-time algorithm $K$ exists which on input $l^{l_{G}}$ outputs $(n, z)$ such that for any probabilistic polynomial-time algorithm $P$, the probability that $P$ can solve the S-RSA Problem is negligible.

\subsection{The Representation Problem [45]}

Definition 1. $k \geq 2$ is polynomial in the length of input, $q$ is a prime. A generator-tuple of length $k$ is $\left(g_{1}, \ldots, g_{k}\right)$, where for $\left.i, j \in\{1, \ldots, k\}, g_{i}, g_{i} \in G_{q} \backslash 1\right\}$, if $i \neq j$, and $g_{i} \neq g_{j}$. An indextuple of length $k$ is $\left(a_{1}, \ldots, a_{k}\right)$ with $a_{i} \in Z_{q}$ for $i \in\{1, \ldots, k\}$. For $h \in G_{q}$, a representation of $h$ with respect to a generator-tuple $\left(g_{1}, \ldots, g_{k}\right)$ is an index-tuple $\left(a_{1}, \ldots, a_{k}\right)$ such that $\prod_{l \leq i \leq k} g_{i}{ }_{i}=h$. The representation $(0, \ldots, 0)$ of $h=1$ is the trivial representing index-tuple.

$\diamond \quad$ The representation problem is to find a representation of $h$ with respect to $\left(g_{1}, \ldots, g_{k}\right)$.

Proposition 1. $G_{q}$ is a group of prime order $q$, and $S$ is the set of all functions of the form $s_{1}(\cdot) / s_{2}(\cdot)$, such that $s_{1}(\cdot)$ and $s_{2}(\cdot)$ are polynomials with integer domain and integer coefficients, and $s_{1}(k)>s_{2}(k) \geq 1$ for all sufficiently large $k$. For any functions $f_{1}(\cdot), f_{2}(\cdot), f_{3}(\cdot)$, $f_{4}(\cdot) \in S:$

(1) There exists a generator-tuple of length $k$ and an algorithm $A_{(1)}$ which, on inputs $h \in G_{q}$, outputs a representing index-tuple of $h$ with probability of success at least $1 / f_{1}(|p|)$ for all $|p|$ large enough.

(2) There exists a generator $g_{k}$ and a polynomial-time algorithm $A_{(2)}$ which, on input a generator-tuple $\left(g_{1}, \ldots, g_{k-1}\right)$, outputs a nontrivial representing index-tuple of 1 with respect to $\left(g_{1}, \ldots, g_{k}\right)$ with probability of success at least $1 / f_{2}(|p|)$ for all $|p|$ large enough.

(3) There exists a $h \in G_{q} \backslash\{1\}$ and a polynomial-time algorithm $A_{(3)}$ which, on inputs a generator-tuple of length $k$, outputs a representing index-tuple of $h$ with probability of success at least $1 / f_{3}(|p|)$ for all $|p|$ large enough. 
(4) There exists a polynomial-time algorithm $A_{(4)}$ which solves the Discrete Log problem with probability of success at least $1 / f_{4}(|p|)$ for all $|p|$ large enough.

The above four statements are equivalent.

So the above Proposition 1 states that in computational difficulty, the representation problem for groups of prime order is equivalent to the Discrete Log problem.

Corollary 1. In a group $G_{q}$ of prime order $q$ and under the Discrete Log assumption, there cannot exist a polynomial-time algorithm which, on input a generator-tuple $\left(g_{1}, \ldots, g_{k}\right)$, $i \in\{1, \ldots, k\}, g_{i} \in_{R} G_{q} \backslash\{1\}$, outputs a number $h \in G_{q}$ and two different representing index-tuples of $h$ with nonnegligible probability.

\subsection{Number-Theoretic Foundations}

Considering the system security, it is necessary to choose a safe RSA modulus $n$, i.e., $n=p q$, with $p \neq q, p=2 p^{\prime}+1, q=2 q^{\prime}+1$, and $p, q, p^{\prime}, q^{\prime}$ are all odd prime. So we can restrict operation to the subgroup of quadratic residues modulo $n$, i.e., the cyclic subgroup $Q R(n)$ generated by an element of order $p^{\prime} q^{\prime}$ which has no small factors. Note that the Corollary 2 not only shows how to achieve the security feature described above, but also provides a method to verify the order of an element without knowing it, which is used in the setup of our system.

Theorem 1. The condition for existence of primitive root modulo $n$ is $n=2,4, p^{\alpha}, 2 p^{\alpha}$, and $\alpha$ is any integer, $p$ is odd prime.

Proposition 2. Let $n=p q$, where $p \neq q, p=2 p^{\prime}+1, q=2 q^{\prime}+1$, and $p, q, p^{\prime}, q^{\prime}$ are all odd prime. Integer a s.t. $\operatorname{gcd}(a, n)=1 . \operatorname{ord}_{n}(a)$, i.e. the order of $a \in Z_{n}{ }^{*}$ is one of the set $\left\{1,2, p^{\prime}, q^{\prime}, 2 p^{\prime}, 2 q^{\prime}, p^{\prime} q^{\prime}, 2 p^{\prime} q^{\prime}\right\}$. Moreover, if ord $\left(\right.$ a) is equal to $p^{\prime} q^{\prime}$ or $2 p^{\prime} q^{\prime} \Leftrightarrow \operatorname{gcd}(a \pm 1, n)=1$.

Corollary 2. Let $p^{\prime}, q^{\prime} \in \in_{R} I_{l p}$ (where $I_{d}$ means $\left.\left.\pm_{\{} 0,1\right\}^{d}\right), n$ be as in Proposition 2. For integer $x$ s.t. $\operatorname{gcd}(x, n)=1$ and $\operatorname{gcd}(x \pm 1, n)=1, a \equiv x^{2}(\bmod n),\langle a\rangle \subset Z_{n}^{*}$ is a cyclic subgroup of order $p^{\prime} q^{\prime}$. Moreover, for $k \in_{R} I_{l p-1}$ s.t. $y \equiv a^{k}(\bmod n),\langle y\rangle \subset Z_{n}^{*}$ is a cyclic subgroup of order $p^{\prime} q^{\prime}$.

\subsection{The Schnorr Identification Protocol [46]}

In the Figure 1, $t$ is the commitment, $c$ is the challenge information, and $s$ is the response. $y$ $\left(=g^{x}\right)$ is published public key of the prover and $x$ is secret key. After performing the protocol, the prover can convince the verifier of his identity by proving his knowledge of the discrete logarithm of $y$. Note that $\in R$ denotes choosing at random.

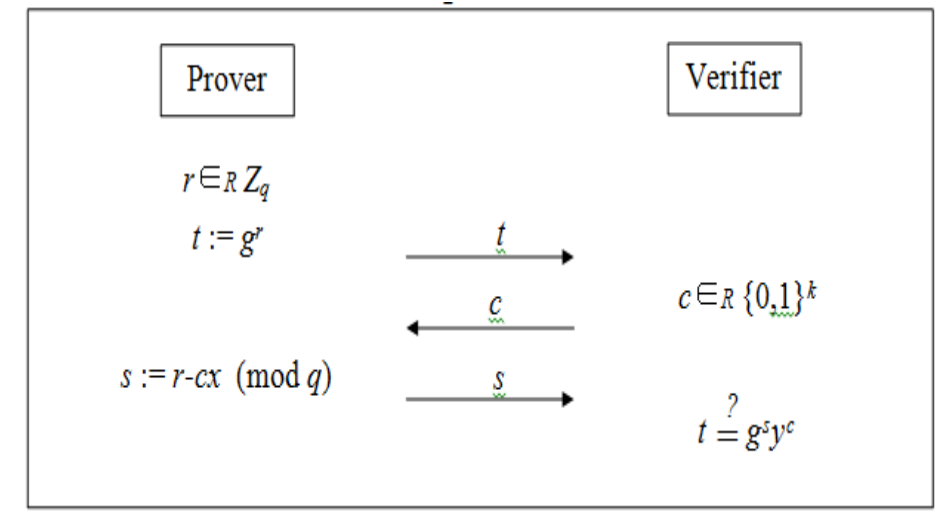

Figure 1. The Schnorr Identification Protocol 
We will see the following signature $\operatorname{SPK}\left(\alpha: y=g^{\alpha}\right)$ can be regarded as the noninteractive version of the Schnorr identification protocol (zero-knowledge proof of $x$ ) by replacing the verifier by a hash function [42].

\subsection{Signatures Based on Proof of Knowledge [9, 10]}

Definition 2. Let $L \in\{0,1\}^{*}$ be a language and let $A=\{A(x)\}_{x \in L}$ and $B=\{B(x)\}_{x \in L}$ be two ensembles of random variables indexed by strings $x \in L . A$ and $B$ are statistically indistinguishable if for every polynomial $p($.) and for all sufficiently long $x \in L$ it holds that $\Sigma$ $\alpha \in\{0,1\}^{*}|\operatorname{Prob}(A(x)=\alpha)-\operatorname{Prob}(B(x)=\alpha)|<1 / p(|x|)$.

Definition 3. An interactive protocol $(P, V)$ is statistical honest-verifier zero-knowledge, if there exists a probabilistic expected polynomial-time simulator $S_{V}$ so that $\{[V, P](x)\}_{x \in L}$ and $\left\{S_{V}(x)\right\}_{x \in L}$ are statistically indistinguishable.

These signatures of knowledge given below can be proved secure in the random oracle model [47], and their interactive versions are statistical (honest-verifier) zero-knowledge proofs of knowledge [11]. The building blocks have in common that the signer does not know the order of $G=\langle g\rangle$, however its bit-length $1_{G}$ (i.e., the integer $l_{G}$ s.t. $2^{l_{G}-1} \leq \# G<2^{l_{G}}$ ) is publicly known. Fujisaki and Okamota show that [42] the standard proofs of knowledge protocols that work for a group of known order are also proofs of knowledge in this setting under the SRSA. We give three basic building blocks: signature of knowledge of (1) a discrete logarithm (Definition 4); (2) equality of two discrete logarithms (Definition 5); (3) a double discrete logarithm (Definition 6). The blocks (1) and (2) will be used in our scheme, and the block (3) will be significantly improved in this paper. $\varepsilon$ is the system security parameter, and $\varepsilon>1$. Finally, let $H:\{0,1\}^{*} \rightarrow\{0,1\}^{\mathrm{k}}$ denote a strong collision-resistant hash function which maps a binary string of arbitrary length to a k-bit hash value.

Definition 4. A pair $(c, s) \in\{0,1\}^{k} \times \pm\{0,1\}^{\left.\varepsilon l_{G}+k\right)+1}$ satisfying $c=H\left(y\|g\| g^{s} y^{c} \| m\right)$ is an $\underline{\boldsymbol{S P K}}$ (signature based on a proof of knowledge) of the discrete logarithm of a group element $y$ to the base $g$, on the message $m \in\{0,1\}^{*}$ and it is denoted as $\operatorname{SPK}\left(\alpha: y=g^{\alpha}\right)(m)$.

The $\operatorname{SPK}\left(\alpha: y=g^{\alpha}\right)(m)$ can be computed if the secret $x=\log _{g} y$ is known, by choosing a random $t \in \pm\{0,1\}^{\left.\varepsilon l_{G}+k\right)}$ and computing $\mathrm{c}$ and s as: $c=H\left(y\|g\| g^{t} \| m\right)$ and $s=t$-cx (in $Z$ ).

Definition 5. A triple $\left(c, s_{1}, s_{2}\right) \in\{0,1\}^{k} \times \pm\{0,1\}^{\varepsilon\left(l_{G}+k\right)+1} \times \pm\{0,1\}^{\varepsilon\left(l_{G}+k\right)+1}$ satisfying $c=$ $H\left(y_{1}\left\|y_{2}\right\| g\|h\| g^{s_{1}} h^{s_{2}} y_{1}{ }^{c}\left\|h^{s_{1}} y_{2}{ }^{c}\right\| m\right)$ is an SPK of secret values $(x, w)$ in $y_{1}=g^{x} h^{w}$ and $y_{2}=h^{x}$ where $y_{1}, y_{2}, g, h \in G$, on a message $m \in\{0,1\}^{*}$ and it is denoted as $\operatorname{SPK}\left(\alpha, \beta: y_{1}=g^{\alpha} h^{\beta} \Lambda\right.$ $\left.y_{2}=h^{\alpha}\right)(m)$.

The $\operatorname{SPK}\left(\alpha, \beta: y_{1}=g^{\alpha} h^{\beta} \wedge y_{2}=h^{\alpha}\right)(m)$ can be computed if the secret $(x, w)$ in $y_{1}=g^{x} h^{w}$ and $y_{2}=h^{x}$ are known, note that $x=\log _{g}\left(y_{1} / h^{w}\right)=\log _{h} y_{2}$, by choosing random pair $\left(t_{1}, t_{2}\right) \in \pm\{0,1\}^{\left.\varepsilon l_{G}+k\right)} \times \pm\{0,1\}^{\left.\varepsilon l_{G}+k\right)}$ and computing $c, \quad s_{1}$ and $s_{2}$ as: $c=H\left(y_{1}\left\|y_{2}\right\| g\|h\| g^{t_{1}} h^{t_{2}}\left\|h^{t_{1}}\right\| m\right), s_{1}=t_{1}-c x$ and $s_{2}=t_{2}-c w$ (all in $Z$ ).

$\diamond \quad$ The Existing SPK of Double Discrete Logarithm

We provide $S P K$ form (cf. [10]) for comparing efficiency later, since the existing knowledge-proof schemes $[10,13,19,27,30-32,35,39]$ usually provide $S P K$ form and describe knowledge proofs in this customary way. As noted earlier, there always exists a corresponding protocol of a zero-knowledge proof of knowledge, i.e., the interactive version of $S P K$. In fact, all the existing $S P K$ schemes of double discrete logarithm have the same mode.

Definition 6. A $(k+1)$ tuple $\left(c, s_{1}, \ldots, s_{k}\right) \in\{0,1\}^{k_{l}} \times \pm\left\{-\left(2^{\lambda}-1\right), \ldots, 2^{\varepsilon \lambda}-1\right\}^{k}$ satisfying $c=H(S\|V\| m)$ with $S=g\|u\| y$ and $V=t_{1}\|\ldots\| t_{k}$, where

for $i=1,2, \ldots, k$, 


$$
\begin{cases}t_{i}=g^{\left(u^{s i}\right)} & \text { if } c[i]=0 \\ t_{i}=y^{\left(u^{s i}\right)} & \text { otherwise, }\end{cases}
$$

is an SPK of a double discrete logarithm $x$ in $y=g^{u^{x}}$, on a message $m \in\{0,1\}^{*}$ ( $\lambda$ is an upper

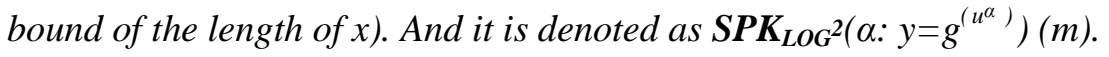

The $\operatorname{SPK}_{L O G^{2}}\left(\alpha: y=g^{u^{a}}\right)(m)$ can be computed as follows if the secret double discrete logarithm $x$ of $y$ to the bases $g$ and $u$ is known, i.e., $x$ in $y=g^{u^{x}}$.

For $i=1,2, \ldots, k$, chooses $r_{i} \in \mathrm{R}\left\{0, \ldots, 2^{\varepsilon \lambda}-1\right\}$ and computes

$$
\tilde{t}_{i}:=g^{\left(u^{r i}\right)}
$$

Then, $c=H\left(g\|u\| y\left\|\tilde{t}_{1}\right\| \tilde{t}_{2}\|\ldots\| \tilde{t}_{k} \| m\right)$, and finally,

is computed for $i=1,2, \ldots, k$.

$$
\begin{cases}s_{i}=r_{i} & \text { if } c[i]=0 \\ s_{i}=r_{i}-x & \text { otherwise, }\end{cases}
$$

It is easy to see that in both computing the proofs and verifying the proofs, the computation complexities of the existing zero-knowledge proof of double discrete logarithm are all $O(k)$. Moreover, the storage complexity and the communication complexity are also $O(k)$. Since in applications, $k>80$, the existing schemes are inefficient.

Note that the knowledge of $u^{x}$ cannot be leaked too when proving the knowledge of $x$ in $y=g^{u^{x}}$, so there is no simple method to achieve zero-knowledge proof of double discrete logarithm. In fact, so far, the efficiency problem of zero-knowledge proof of double discrete logarithm has not been solved.

\section{New Zero-knowledge Proof of Knowledge of Double Discrete Logarithm}

\subsection{Basic Construction}

Definition 7. A triple $\left(c, s, s^{\prime}\right) \in\{0,1\}^{k} \times\left\{-\left(2^{l_{G}}+2^{k}-2\right), \ldots, 2^{\varepsilon\left(l_{G}+2\right)}-1\right\} \times\left\{-\left(2^{k}-1\right)\left(2^{\varepsilon\left(l_{G}+2\right)}-\right.\right.$ 1), $\left.\ldots, 2^{\varepsilon\left[\varepsilon\left(l_{G}+2\right)+k\right]}-1\right\}$ satisfying $c=H\left(y\|g\| y g^{c+s}\left\|y^{c} g^{c^{2}+c s+s^{\prime}}\right\| m\right)=H\left(y\|g\| y g^{c+s}\left\|\left(y g^{c+s}\right)^{c} g^{s^{\prime}}\right\| m\right)$ is an SPK of the discrete logarithm of a group element $y$ to the base $g$, on the message $m \in\{0,1\}^{*}$ and it is denoted as $\operatorname{SPK}_{\text {LOGnew }}\left(\alpha: y=g^{\alpha}\right)(m)$.

In our new SPK system, the security parameter of system $\varepsilon>1, y=g^{x}(\bmod n)$ with $x \in\{0,1\}^{l_{G}}$, and $l_{G}>k$. The $S P K_{\text {LOGnew }}\left(\alpha: y=g^{\alpha}\right)(m)$ can be computed if the secret $x$ is known, by choosing a random $\left(t, t^{\prime}\right) \in\{0,1\}^{\varepsilon\left(l_{G}+2\right)} \times\{0,1\}^{\varepsilon\left[\varepsilon\left(l_{G}+2\right)+k\right]}$ and computing $c, s, s^{\prime}$ as:

$$
\begin{aligned}
& \Delta=g^{t}(\bmod n) \\
& \Delta^{\prime}=g^{t^{\prime}}(\bmod n) \\
& c=H\left(y\|g\| \Delta\left\|\Delta^{\prime}\right\| m\right) \\
& s=t-x-c \quad(\text { in Z }) \\
& s^{\prime}=t^{\prime}-c t(\text { in Z })
\end{aligned}
$$

It seems that there is one more modular exponentiation in $S P K_{\text {LOGnew }}$ comparing with conventional signatures of knowledge, but it will be proved essential to our system. That is to say, $S P K_{L O G n e w}$ plays an important role in the proofs of knowledge of double discrete logarithm. In fact, we will see that it significantly improves the efficiency of proofs of knowledge of double discrete logarithm. The security proofs of $S P K_{L O \text { Gnew }}$ are provided as follows.

Theorem 2. Under the Discrete Logarithm assumption, If the signer uses the knowledge of $x$ to complete $S P K_{\text {LOGnew, }}$ it follows that the signer has knowledge of $x$ satisfying $y=g^{x}(\bmod n)$.

Proof. We take two steps to prove that the signer has the knowledge of $x$ satisfying $y=g^{x}(\bmod n)$. 
Step 1: Prove $y^{c} g^{c^{2}+c s+s^{\prime}}$ in the verifying equation $c=H\left(\right.$ ) is a $\operatorname{SPK}\left(\alpha: y g^{c+s}=g^{\alpha}\right)$.

It is sufficient to show that the knowledge extractor can compute the witness once he has found two accepting quintuples $\left(c, s, s^{\prime}, \Delta=g^{t}, \Delta^{\prime}=g^{t^{\prime}}\right)$ and $\left(\hat{c}, \hat{s}, \hat{s}^{\prime}, \Delta=g^{t}, \Delta^{\prime}=g^{t^{\prime}}\right)$.

When the signer complete $S P K_{\text {LOGnew }}, s=t-x-c$ (in Z), $\hat{s}=t-x-\hat{c}$ (in Z) $\Rightarrow c+s=\hat{c}+\hat{s}=$ $t-x$ (in Z).

So when the knowledge extractor works, $\Delta^{\prime}=y^{c} g^{c^{2}+c s+s^{\prime}}=\left(y g^{c+s}\right)^{c} g^{s^{\prime}}=\left(y g^{\hat{c}+\hat{s}}\right)^{\hat{c}} g^{\hat{s}^{\prime}}=$ $y^{\hat{c}} g^{\hat{c}^{2}+\hat{c} \hat{s}+\hat{s}^{\prime}}(\bmod n) \Rightarrow\left(y g^{c+s}\right)^{c} g^{s^{\prime}}=\left(y g^{c+s}\right)^{\hat{c}} g^{\hat{s}^{\prime}}(\bmod n) . g$ is chosen satisfying $\operatorname{gcd}(n, g)=1$, and $\operatorname{gcd}(n, y)=1$ that can be verified in advance $\Rightarrow\left(y g^{c+s}\right)^{c-\hat{c}}=g^{\hat{s}^{\prime}-s^{\prime}}(\bmod n)$.

Let $d:=\operatorname{gcd}\left(c-\hat{c}, \hat{s}^{\prime}-s\right)$. $t_{1}$ and $t_{2}$ can be obtained by using the extended Euclidean algorithm, which are satisfying

So

$$
\begin{aligned}
& t_{1} \cdot(c-\hat{c}) / d+t_{2} \cdot\left(\hat{s}^{\prime}-s\right) / d=1 \\
& g \quad=g^{t_{1} \cdot(c-\hat{c}) / d+t_{2} \cdot\left(\hat{s}^{\prime}-s\right) / d} \\
& =g^{t_{1} \cdot(c-\hat{c}) / d} \cdot\left(y g^{c+s}\right)^{t_{2} \cdot(c-\hat{c}) / d} \\
& =\left[g^{t_{1}} \cdot\left(y g^{c+s}\right)^{t_{2}}\right]^{(c-\hat{c}) / d}
\end{aligned}
$$

If $d<c$ - $\hat{c}$, we get $\left[g^{t_{1}} \cdot\left(y g^{c+s}\right)^{t_{2}}\right]$ is $(c-\hat{c}) / d$-root of $g$. But because it contradicts Assumption 1, we must have $d=c$ - $\hat{c}$, hence $c$ - $\hat{c}$ divides $\hat{s}^{\prime}-s^{\prime}$.

$y g^{c+s}=g^{\alpha}(\bmod n)$ which the signer uses to complete $S P K_{\text {LOGnew }}$, and $\left(y g^{c+s}\right)^{c-\hat{c}}=g^{\hat{s}^{\prime}-s^{\prime}}$ $(\bmod n)$ which is deduced above $\Rightarrow g^{\alpha(c-\hat{c})}=g^{\hat{s}^{\prime}-s^{\prime}}(\bmod n), \operatorname{gcd}(n, g)=1 \Rightarrow g^{\alpha}=g^{\left(\hat{s}^{\prime}-s\right) /(c-\hat{c})}$ $(\bmod n) \Leftrightarrow \alpha \equiv\left(\hat{s}^{\prime}-s\right) /(c-\hat{c})\left(\bmod \operatorname{ord}_{n}(g)\right)$

So the knowledge extractor can compute $\alpha$ without the knowledge of $\operatorname{ord}_{n}(g)$ :

$\alpha:=\left(\hat{s}^{\prime}-s\right) /(c-\hat{c})$ such that $y g^{c+s}=g^{\alpha}(\bmod n)$. So the signer has the knowledge of $\alpha$ satisfying $y g^{c+s}=g^{\alpha}(\bmod n)$.

Step 2: Analyze whether $s$ is generated before $c$ or not. Suppose $s$ is generated before $c$. Then $\Delta=y g^{c+s}(\bmod n)$ which is in $H\left(y\|g\| \Delta\left\|\Delta^{\prime}\right\| m\right)$ for $H()$ is a strong collision-resistant function and solving the representation problem (see in the Definition 1) is considered infeasible. $\Delta=y g^{c+s}=\left(y g^{s}\right) g^{c}$, as $y$ is fixed and $s$ is supposed generated before $c, \Delta$ is determined by $g^{c} . c$ is determined before $g^{c}$ for the Discrete Logarithm assumption, so $c$ is determined before $\Delta$ which is a part of input of $H($ ). It contradicts that $H()$ is a one-way function, so $s$ is not generated before $c$. For the same reason, $s$ and $c$ are not generated at the same time.

So $s$ is generated after $c$, and according to the result of Step $1, y g^{c+s}=g^{\alpha}(\bmod n) \Rightarrow$ $s=\log _{g}\left(g^{\alpha} / y\right)-c$. As a result, $\log _{g}\left(g^{\alpha} / y\right)$ has to be computed. If the signer has no knowledge of $x$ satisfying $y=g^{x}(\bmod n)$, it contradicts the Discrete Logarithm assumption.

So it can conclude that the signer has the knowledge of $x$ satisfying $y=g^{x}(\bmod n)$.

Theorem 3. Under the Discrete Logarithm assumption, the probability for the signer without discrete logarithm knowledge of y to generate a valid signature is less than $2^{-k}$.

Proof. Obviously if the signer $S$ follows the protocol then the verifier $V$ always accepts the signature. Let $\tilde{S}$ denotes a fraudulent $S$. $\tilde{S}$ can deviate from the protocol. We next consider the possibilities of cheating for $\tilde{S}$, i.e., $\tilde{S}$ does not know $x$ and can generate a signature which can be verified by anyone. Note that in our signature scheme, $m$ may contain any random element which is provided by $V$, that is to say, the randomness of $c$ can be provided by $S$ and $V$ jointly.

Under the Discrete Logarithm assumption, $x$ can not be achieved from $y$, so $\tilde{S}$ can not finish the signature following the normal way. It concludes that $S P K_{\text {LOGnew }}$ consists of $\operatorname{SPK}\left(\alpha: y g^{c+s}=g^{\alpha}\right)$ and $\operatorname{SPK}\left(\alpha: y=g^{\alpha}\right)$ in the proof of Theorem 2. If $\tilde{S}$ has no knowledge of discrete logarithm of $y$ to the base $g$, he can not complete $\operatorname{SPK}\left(\alpha: y g^{c+s}=g^{\alpha}\right)$ either, 
otherwise it contradicts the Discrete Logarithm assumption. So for cheating, $\tilde{S}$ has to guess the right triple $\left(c, s, s^{\prime}\right) \in\{0,1\}^{k} \times\left\{-\left(2^{l_{G}}+2^{k}-2\right), \ldots, 2^{\varepsilon\left(l_{G}+2\right)}-1\right\} \times\left\{-\left(2^{k}-1\right)\left(2^{\varepsilon\left(l_{G}+2\right)}-\right.\right.$ 1) $\left., \ldots, 2^{\varepsilon\left[\varepsilon\left(l_{G}+2\right)+k\right]}-1\right\}$.

$\tilde{S}$ can choose arbitrary $\left(s_{r}, s_{r}{ }^{\prime}\right) \in\left\{-\left(2^{l_{G}}+2^{k}-2\right), \ldots, 2^{\varepsilon\left(l_{G}+2\right)}-1\right\} \times\left\{-\left(2^{k}-1\right)\left(2^{\varepsilon\left(l_{G}+2\right)}-\right.\right.$ $\left.1), \ldots, 2^{\varepsilon\left[\varepsilon\left(l_{G}+2\right)+k\right]}-1\right\}$, and then guess the correct $c$ by Algorithm $_{\text {guess }}$ :

(1) count $\in Z$, count $=1$;

(2) If count $=1$, choose different $\left(s_{r}, s_{r}{ }^{\prime}\right) \in\left\{-\left(2^{l_{G}}+2^{k}-2\right), \ldots, 2^{\varepsilon\left(l_{G}+2\right)}-1\right\} \times\left\{-\left(2^{k}-1\right)\left(2^{\varepsilon\left(l_{G}+2\right)}-\right.\right.$ 1) $\left., \ldots, 2^{\varepsilon\left[\varepsilon\left(l_{G}+2\right)+k\right]}-1\right\}$;

(3) Uniformly choose a different $c_{g} \in\{0,1\}^{k}$ at random;

(4) $\Delta:=y g^{c_{g}+s_{r}}, \Delta^{\prime}:=y^{c_{g}} g^{c_{g}{ }^{2}+c_{g} s_{r}+s_{r_{r}}}(\bmod n)$;

(5) $c=H\left(y\|g\| \Delta\left\|\Delta^{\prime}\right\| m\right)$, count $=$ count +1 ;

(6) When $c_{g} \neq c$, if count $\leq 2^{k}$, go to (3);

if count $=2^{k}+1$, go to (1);

Otherwise go to (7);

(7) $c:=c_{g}, s:=s_{r}, s^{\prime}:=s_{r}{ }^{\prime}$. Output $\left(c, s, s^{\prime}\right)$.

So the triple $\left(c, s, s^{\prime}\right) \in\{0,1\}^{k} \times\left\{-\left(2^{l_{G}}+2^{k}-2\right), \ldots, 2^{\varepsilon\left(l_{G}+2\right)}-1\right\} \quad \times \quad\left\{-\left(2^{k}-1\right)\left(2^{\varepsilon\left(l_{G}+2\right)}-\right.\right.$ 1), $\left.\ldots, 2^{\varepsilon\left[\varepsilon\left(l_{G}+2\right)+k\right]}-1\right\}$ satisfying $c=H\left(y\|g\| y g^{c+s}\left\|y^{c} g^{c^{2}+c s+s^{\prime}}\right\| m\right)$ is the crooked proof.

$\tilde{S}$ has no advantage in guessing $c$ in the scheme. First, as mentioned above, the randomness of $c$ is provided by $S$ and $V$ jointly. Moreover, $H()$ is a strong collisionresistant and one-way hash function, and $c$ can not be generated before choosing $c_{g}$. As a result, $\tilde{S}$ has to uniformly choose $c_{g} \in\{0,1\}^{k}$ and then test it. So the probability of success for this attack Prob success $<2^{-k}$, and therefore this attack becomes infeasible for sufficiently large $k$, e.g. $k=160$.

There are two reasons why choose $\left(s_{r}, s_{r}{ }^{\prime}\right)$ before $c_{g}$ in Algorithm $_{\text {guess }}$. First, we can analyze the probability distribution of the correct $\left(s, s^{\prime}\right)$ in $S P K_{\text {LOGnew }}$ (see it in the proof of Theorem 4), which is helpful for choosing $s$. Second, as $l_{G}>k,\{0,1\}^{k}$ is a subset of any of $\left\{-\left(2^{l_{G}}+2^{k}-2\right), \ldots, 2^{\varepsilon\left(l_{G}+2\right)}-1\right\}$ and $\left\{-\left(2^{k}-1\right)\left(2^{\varepsilon\left(l_{G}+2\right)}-1\right), \ldots, 2^{\varepsilon\left[\varepsilon\left(l_{G}+2\right)+k\right]}-1\right\}$, so it can conclude Prob $_{\text {most }}$ success $_{2}<2^{-k}$ rather than Prob sust $_{\text {sucess }}<\mu$ with $\mu=\left[\left(2^{l_{G}}+2^{k}-2\right)+2^{\varepsilon\left(l_{G}+2\right)}-1\right]^{-1}$ or $\mu=\left\{\left(2^{k}-1\right)\left(2^{\varepsilon\left(l_{G}+2\right)}-1\right)+2^{\varepsilon\left[\varepsilon\left(l_{G}+2\right)+k\right]}-1\right\}^{-1}$, where $\mu<2^{-k}$, i.e., the upper limit of the probability of success for this attack is $2^{-k}$.

We explain why there is the possibility of going to step (1) in step (6) in Algorithm $_{\text {guess. }}$.

The correct triple $\left(c, s, s^{\prime}\right)$ is:

$$
\begin{aligned}
& c=H\left(y\|g\| g^{t}(\bmod n)\left\|g^{t^{\prime}}(\bmod n)\right\| m\right) \ldots \text { (1) } \\
& s=t-x-c(\text { in Z }) \\
& s^{\prime}=t^{\prime}-c t(\text { in Z })
\end{aligned}
$$

Observe $t \in\{0,1\}^{\varepsilon\left(l_{G}+2\right)}, t^{\prime} \in\{0,1\}^{\varepsilon\left[\varepsilon\left(l_{G}+2\right)+k\right]}, x \in\{0,1\}^{l_{G}}, c \in\{0,1\}^{k}$, and we can conclude $s \in\left\{-\left(2^{l_{G}}+2^{k}-2\right), \ldots, 2^{\varepsilon\left(l_{G}+2\right)}-1\right\}$ and $s^{\prime} \in\left\{-\left(2^{k}-1\right)\left(2^{\varepsilon\left(l_{G}+2\right)}-1\right), \ldots, 2^{\varepsilon\left[\varepsilon\left(l_{G}+2\right)+k\right]}-1\right\}$ from (2) and (3) . Note that the ranges are related to the probability distribution of important system parameters. But $c=H\left(y\|g\| g^{t}(\bmod n)\left\|g^{t^{\prime}}(\bmod n)\right\| m\right)$, i.e., $c$ is a function $\mathrm{f}\left(y, t, t^{\prime}, m\right)$, and $\mathrm{f}\left(y, t, t^{\prime}, m\right)$ is a one-way function, so $c$ in (2) and (3) can not be chosen arbitrarily, thus $\left(s, s^{\prime}\right)$ can not traverse $\left\{-\left(2^{l_{G}}+2^{k}-2\right), \ldots, 2^{\varepsilon\left(l_{G}+2\right)}-1\right\} \times\left\{-\left(2^{k}-1\right)\left(2^{\varepsilon\left(l_{G}+2\right)}-1\right), \ldots, 2^{\varepsilon\left[\varepsilon\left(l_{G}+2\right)+k\right]}-\right.$ $1\}$, even $x$ and $m$ are not particular ones. As a result, the random selection of $\left(s_{r}, s_{r}{ }^{\prime}\right)$ may lead Algorithm guess $_{1}$ to choose it again, though $t, t^{\prime}$ can vary in their ranges arbitrarily. 
$C\left(S, S^{\prime}\right.$, resp.) denotes guessing $c$ (choosing $s, s^{\prime}$, resp.) correctly. For $C, S$ and $S^{\prime}$ are independent events for the ones without any knowledge of $y, \operatorname{Prob}\left(S \cap S^{\prime} \cap C\right)=$ $\operatorname{Prob}(S \cap S) \cdot \operatorname{Prob}(C)=\operatorname{Prob}\left(S \cap S^{\prime}\right) \cdot 2^{-k}<2^{-k}$.

Theorem 4. Under the Discrete Logarithm assumption, the interactive protocol corresponding to $S_{P} K_{\text {LOGnew }}\left(\alpha: y=g^{\alpha}\right)(m)$ is an honest-verifier statistical zeroknowledge proof of knowledge of the discrete logarithm of $y$.

Proof. To prove that the scheme is statistical honest-verifier zero-knowledge for $\varepsilon>1$, we have to show that an honest verifier, i.e., the one who gives the challenge $c \in$ $\{0,1\}^{k}$ uniformly, can simulate a protocol-conversation that is statistically indistinguishable from a protocol-conversation with the signer. The following constitutes a simulator the verifier could use to do so.

The simulator randomly chooses $\bar{s}$ from $\left\{-\left(2^{l_{G}}+2^{k}-2\right), \ldots, 2^{\varepsilon\left(l_{G}+2\right)}-1\right\}, \bar{s}^{\prime}$ from $\left\{-\left(2^{k}-\right.\right.$ 1) $\left.\left(2^{\varepsilon\left(l_{G}+2\right)}-1\right), \ldots, 2^{\varepsilon\left[\varepsilon\left(l_{G}+2\right)+k\right]}-1\right\}$ and $\bar{c}$ from $\{0,1\}^{k}$ according to the uniform distribution. Using these values, the simulator computes $\bar{\Delta}=y g^{\bar{c}+\bar{s}}(\bmod n)$, and $\overline{\Delta^{\prime}}=y^{\bar{c}} g^{\bar{c}+\overline{c s s}+\bar{s}^{\prime}}$. To prove that these values are statistical indistinguishable from a view of a protocol run with the signer, it suffices to consider the probability distribution $P_{S}(s)$ of the response $s$ and $P_{S^{\prime}}\left(s^{\prime}\right)$ of the response $s^{\prime}$ of the signer and the probability distribution $P_{\bar{S}}(\bar{s})$ and $P_{\bar{S}^{\prime}}\left(\bar{s}^{\prime}\right)$ according to which the simulator choose $\bar{s}$ and $\bar{s}^{\prime} . \bar{s}$ is the uniform distribution over $\left\{-\left(2^{l_{G}}+2^{k}-2\right), \ldots, 2^{\varepsilon\left(l_{G}+2\right)}-1\right\}$, and $\bar{s}^{\prime}$ is the uniform distribution over $\left\{-\left(2^{k}-1\right)\left(2^{\varepsilon\left(l_{G}+2\right)}-\right.\right.$ 1), .., $\left.2^{\varepsilon\left[\varepsilon\left(l_{G}+2\right)+k\right]}-1\right\}$.

In the $S P K_{\text {LOGnew }}$, the signer chooses $t$ uniformly at random from $\{0,1\}^{\varepsilon\left(l_{G}+2\right)}, t^{\prime}$ uniformly at random from $\{0,1\}^{\varepsilon\left[\varepsilon\left(l_{G}+2\right)+k\right]}$ and the secret key $x$ randomly from $\{0,1\}^{l_{G}}$ according to any distribution, then we have

$$
\begin{aligned}
& P_{S}(s) \quad \begin{cases}=0 & \text { for } s<-\left(2^{l_{G}}+2^{k}-2\right) \\
\leq 2^{-\varepsilon\left(l_{G}+2\right)} & \text { for }-\left(2^{l_{G}}+2^{k}-2\right) \leq s<0 \\
=2^{-\varepsilon\left(l_{G}+2\right)} & \text { for } 0 \leq s \leq 2^{\varepsilon\left(l_{G}+2\right)}-1-\left(2^{l_{G}}+2^{k}-2\right) \\
\leq 2^{-\varepsilon\left(l_{G}+2\right)} & \text { for } 2^{\varepsilon\left(l_{G}+2\right)}-1-\left(2^{l_{G}}+2^{k}-2\right)<s \leq 2^{\varepsilon\left(l_{G}+2\right)}-1 \\
=0 & \text { for } 2^{\varepsilon\left(l_{G}+2\right)}-1<s \\
=0 & \text { for } s^{\prime}<-\left(2^{k}-1\right)\left(2^{\varepsilon\left(l_{G}+2\right)}-1\right)\end{cases} \\
& P_{S^{\prime}}\left(s^{\prime}\right) \quad \begin{cases}\leq 2^{-\varepsilon\left[\varepsilon\left(l_{G}+2\right)+k\right]} & \text { for }-\left(2^{k}-1\right)\left(2^{\varepsilon\left(l_{G}+2\right)}-1\right) \leq s^{\prime}<0 \\
=2^{-\varepsilon\left[\varepsilon\left(l_{G}+2\right)+k\right]} & \text { for } 0 \leq s^{\prime} \leq 2^{\left.\varepsilon \varepsilon\left(l_{G}+2\right)+k\right]}-1-\left(2^{k}-1\right)\left(2^{\varepsilon\left(l_{G}+2\right)}-1\right) \\
\leq 2^{-\varepsilon\left[\varepsilon\left(l_{G}+2\right)+k\right]} & \text { for } 2^{\varepsilon\left[\varepsilon\left(l_{G}+2\right)+k\right]}-1-\left(2^{k}-1\right)\left(2^{\varepsilon\left(l_{G}+2\right)}-1\right)<s^{\prime} \leq 2^{\varepsilon\left[\varepsilon\left(l_{G}+2\right)+k\right]}-1 \\
=0 & \text { for } 2^{\varepsilon\left[\varepsilon\left(l_{G}+2\right)+k\right]}-1<s^{\prime}\end{cases}
\end{aligned}
$$

We give a brief explanation of the above results. For example,

$P(s=0)=\sum P(t-x-c=0)=\sum P(t=x+c)=P(t=0, x+c=0)+P(t=1, x+c=1)+\ldots+P\left(t=2^{\varepsilon\left(l_{G}+2\right)}-1\right.$, $\left.x+c=2^{\varepsilon\left(l_{G}+2\right)}-1\right)$

Because $t$ and $x$ are mutually independent random variables, and $c$ is the output of random oracle model, and $t$ is the uniform distribution over $\left\{0, \ldots, 2^{\varepsilon\left(l_{G}+2\right)}-1\right\}$,

$$
P(s=0)=2^{-\varepsilon\left(l_{G}+2\right)} \cdot\left[P(x+c=0)+P(x+c=1)+\ldots+P\left(x+c=2^{\varepsilon\left(l_{G}+2\right)}-1\right)\right]=2^{-\varepsilon\left(l_{G}+2\right)} \cdot[P(x+c=0)+
$$
$\left.P(x+c=1)+\ldots+P\left(x+c=2^{l_{G}}+2^{k}-2\right)+0\right]=2^{-\varepsilon\left(l_{G}+2\right)} \cdot 1=2^{-\varepsilon\left(l_{G}+2\right)}$

So this holds for any distribution of $c$ over $\{0,1\}^{k}$. The figure 2 illustrates the distribution of $P_{\bar{S}}(\bar{S})$ and $P_{S}(s)$, and the distribution of $P_{\bar{S}^{\prime}}\left(\bar{S}^{\prime}\right)$ and $P_{S^{\prime}}\left(s^{\prime}\right)$ which we omit is similar to it, thus we have

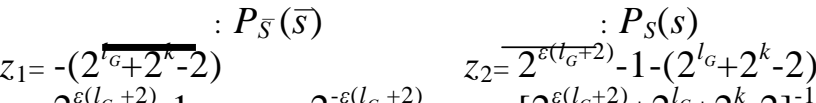

$$
\begin{aligned}
& z_{3}=2^{\varepsilon\left(l_{G}+2\right)}-1 \quad z_{4}=2^{-\varepsilon\left(l_{G}+2\right)} \quad z_{5}=\left[2^{\varepsilon\left(l_{G}+2\right)}+2^{l_{G}}+2^{k}-2\right]^{-1}
\end{aligned}
$$




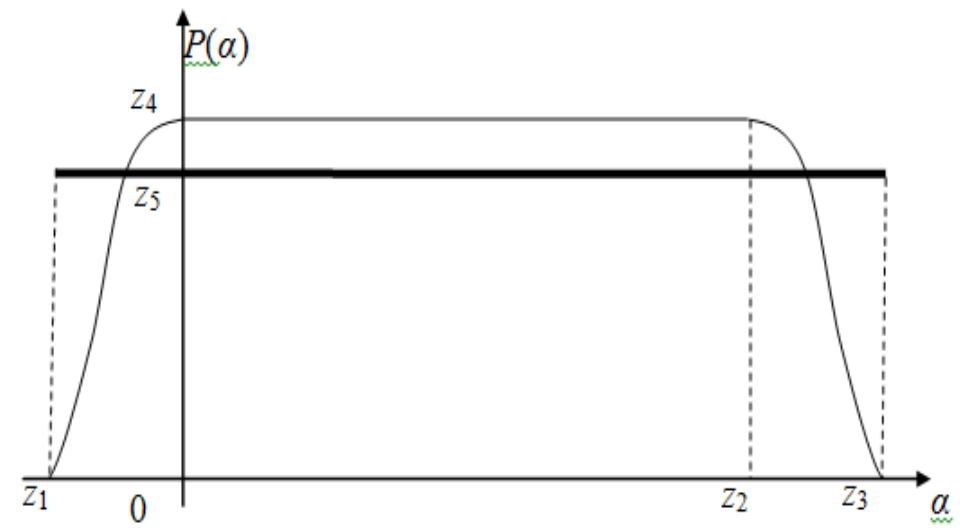

Figure 2. The Distribution of $P_{\alpha}(\alpha)$

$$
\begin{aligned}
& \left.\sum_{\alpha \in \mathrm{Z}}\left|P_{S}(\alpha)-P_{S}(\alpha)\right| \underset{\alpha \in\left\{-\left(2^{1 G}+2^{k}-2\right), \ldots, 2^{\varepsilon(G+2)}-1\right\}}{=} \mid P^{\varepsilon\left(l_{G}+2\right)}+2^{l_{G}}+2^{k}-2\right] \mid \\
& \leq\left\{1 / 2^{\varepsilon\left(l_{G}+2\right)}-1 /\left[2^{\varepsilon\left(l_{G}+2\right)}+2^{l_{G}}+2^{k}-2\right]\right\}\left[2^{\varepsilon\left(l_{G}+2\right)}-2^{l_{G}}-2^{k}+2\right]+2\left(2^{l_{G}}+2^{k}-2\right) 2^{\varepsilon\left(l_{G}+2\right)} \\
& \leq\left(2^{l_{G}}+2^{k}-2\right) 2^{\varepsilon\left(l_{G}+2\right)}+2\left(2^{l_{G}}+2^{k}-2\right) / 2^{\varepsilon\left(l_{G}+2\right)}=3\left(2^{l_{G}}+2^{k}-2\right) / 2^{\varepsilon\left(l_{G}+2\right)} \\
& \leq 4\left(2^{l_{G}+1}-2\right) 2^{\varepsilon\left(l_{G}+2\right)} \leq 2^{l_{G}+3} / 2^{\varepsilon\left(l_{G}+2\right)} \leq 2^{l_{G}+2 \varepsilon} / 2^{\varepsilon l_{G}+2 \varepsilon} \leq 1 / 2^{(\varepsilon-1) l_{G}} \\
& \sum\left|P_{S^{\prime}}(\alpha)-P_{\bar{S}^{\prime}}(\alpha)\right|=\sum\left|P_{S^{\prime}}(\alpha)-\left[2^{\varepsilon\left[\varepsilon\left(l_{G}+2\right)+k\right]}+\left(2^{k}-1\right)\left(2^{\varepsilon\left(l_{G}+2\right)}-1\right)\right]^{-1}\right| \\
& \alpha \in Z \quad \quad \alpha \in\left\{-\left(2^{k}-1\right)\left(2^{\varepsilon(G)+2)}-1\right), \ldots, 2^{\varepsilon[\varepsilon(G+2)+k]}-1\right\} \\
& \leq\left\{2^{-\varepsilon\left[\varepsilon\left(l_{G}+2\right)+k\right]}-\left[2^{\varepsilon\left[\varepsilon\left(l_{G}+2\right)+k\right]}+\left(2^{k}-1\right)\left(2^{\varepsilon\left(l_{G}+2\right)}-1\right)\right]^{-1}\right\} \cdot\left[2^{\varepsilon\left[\varepsilon\left(l_{G}+2\right)+k\right]}-\left(2^{k}-1\right) \cdot\left(2^{\varepsilon\left(l_{G}+2\right)}-1\right)\right] \\
& +2\left(2^{k}-1\right)\left(2^{\varepsilon\left(l_{G}+2\right)}-1\right) \cdot 2^{-\varepsilon\left[\varepsilon\left(l_{G}+2\right)+k\right]} \\
& \leq\left(2^{k}-1\right)\left(2^{\varepsilon\left(l_{G}+2\right)}-1\right) \cdot 2^{-\varepsilon\left[\varepsilon\left(l_{G}+2\right)+k\right]}+2\left(2^{k}-1\right)\left(2^{\varepsilon\left(l_{G}+2\right)}-1\right) \cdot 2^{-\varepsilon\left[\varepsilon\left(l_{G}+2\right)+k\right]} \\
& =3\left(2^{k}-1\right)\left(2^{\varepsilon\left(l_{G}+2\right)}-1\right) \cdot 2^{-\varepsilon\left[\varepsilon\left(l_{G}+2\right)+k\right]} \leq 2^{\varepsilon\left(l_{G}+2\right)+k+2} \cdot 2^{-\varepsilon\left[\varepsilon\left(l_{G}+2\right)+k\right]}=1 / 2^{(\varepsilon-1)\left[\varepsilon\left(l_{G}+2\right)+k\right]-2}
\end{aligned}
$$

For $\varepsilon>1$ and $l_{G}>k=160$, the denominators of last terms of the above computing can be expressed as one over a polynomial in the input length, therefore the distributions of $s$ and $\bar{s}$ are statistical indistinguishable, also the distributions of $s^{\prime}$ and $\bar{s}^{\prime}$ are statistical indistinguishable.

So the interactive protocol corresponding to $S P K_{\text {new }}\left(\alpha: y=g^{\alpha}\right)(m)$ is an honest-verifier statistical zero-knowledge proof according to Definition 3. (We can see that $\varepsilon$ controls the tightness of the statistical zero-knowledge.)

The idea of our proofs for Theorem 4 is inspired by [11], and the method of which derives from [46].

\subsection{New Zero-knowledge Proof of Knowledge of Double Discrete Logarithm}

TTP's Setup:

$\diamond$ Select random secret $l_{p}$-bits primes $p^{\prime \prime}, q^{\prime \prime}$ such that $p^{\prime}=2 p^{\prime \prime}+1, q^{\prime}=2 q^{\prime \prime}+1, p=$ $2 p^{\prime}+1$ and $q=2 q^{\prime}+1$ are all primes. Provide a proof that $n=p q$ is the product of two safe primes [12].

$\diamond \quad$ Choose random $Q R(n) g$ with order $p^{\prime} q^{\prime}$ and a prime $u$ with order $p^{\prime \prime} q^{\prime \prime}$ (which can be verified without knowing it, see Corollary 2).

$\diamond \quad$ Compute $y_{\mathrm{T}}=h^{x_{\mathrm{T}}}$ with secret key $x_{\mathrm{T}} \in_{R}\{0,1\}^{l_{G}}$.

The system public parameters are $\left(g, h, n, p^{\prime} q^{\prime}\right)$, and the secret parameters $\left(p^{\prime \prime}, q^{\prime \prime}\right)$ are not kept. Note that the TTP is not indispensable for all our schemes. We provide three schemes of knowledge proof of double discrete logarithm. 
The prover convinces a verifier that he knows the secret double discrete logarithm $x$ in $y=g^{u^{x}}(\bmod n)$ or $\left(x_{1}, x_{2}, \ldots, x_{i}\right)$ in $y=g^{u_{1}{ }^{x} u_{2} x_{2} \ldots u_{i}^{x_{i}}}(\bmod n)$. Note that $i \in \mathrm{Z}$ and $x \in_{R}\{0,1\}^{l_{G}}$, where $I_{d}= \pm\{0,1\}^{d}, \in_{R}$ denotes choosing at random.

$>$ Without the TTP, to achieve this, the prover does as follows.

Protocol $_{\underline{L O G}}{ }^{2} \_1$ :

(1) The prover chooses $r \in \in_{R}\{0,1\}^{\varepsilon\left(l_{G}+2\right)}$ which has factor 6 , then computes commitment $A=g^{u^{r}}(\bmod n)$ and sends $y$ and $A$ to the verifier;

(2) The verifier chooses the first challenge information $m \in{ }_{R}\{0,1\}^{\varepsilon\left(l_{G}+2\right)}$, and then sends $m$ to the prover;

(3) The prover computes $S P K_{A}=\operatorname{SPK}\left(\alpha, \beta: A=g^{\alpha^{2}}=g^{\beta^{3}}\right)(m)$. Then the prover sends $S P K_{A}$ to the verifier;

(4) The verifier verifies $S P K_{A}$, chooses the second challenge information $c \in_{R}\{0,1\}^{k}$ and sends it to the prover;

(5) The prover computes the $s=r-x-c$ and send s to the verifier;

(6) The verifier verifies $A=y^{u^{c+s}}(\bmod n)$.

If the prover and the verifier follow the protocol then the verifier always accepts the prover's proof of knowledge of double discrete logarithm. We give the proof with the following Theorem 5 .

Theorem 5. Under the Discrete Logarithm assumption and the S-RSA assumption, the interactive protocol Protocol LOG__l $_{2}$ is an honest-verifier statistical zero-knowledge proof of knowledge of the double discrete logarithm of $y=g^{u^{x}}(\bmod n)$ or knowledge proof of knowledge of the double discrete logarithm of $y=g^{u_{1}{ }^{x_{1}}{ }_{2}{ }_{2} \ldots u_{i}^{x_{i}}}(\bmod n)$.

Proof.

According to the $S P K_{A}$ in the step (3) of $\operatorname{Protocol}_{L_{O G}{ }_{-}}$, we have $A=g^{\alpha^{2}}=g^{\Delta_{1}}(\bmod n)$ with some known $\Delta_{1}$.

In the step (6) of Protocol $_{L_{O G}}{ }_{1} 1, A=y^{u^{c+s}}(\bmod n)$. So $y=A^{\left(u^{c+s}\right)^{-1}}=g^{\Delta_{1} \cdot\left(u^{c+s}\right)^{-1}}=g^{\Delta_{2}}(\bmod n)$.

From $A=y^{u^{c+s}}(\bmod n)$ in the step (6) of $\operatorname{Protocol}_{L O G^{2}}{ }_{1}$, we have $\Delta_{1}=\Delta_{2} \cdot u^{c+s}\left(\bmod _{\operatorname{ord}_{n}}(g)\right)$ $=\Delta_{2} \cdot u^{c+s}\left(\bmod p^{\prime} q^{\prime}\right)$, so we have $\Delta_{1}=\Delta_{3} \cdot u^{r}\left(\bmod p^{\prime} q^{\prime}\right)$. That is to say,

$\Delta_{1}=\Delta_{3} \cdot u^{r}=\Delta_{2} \cdot u^{c+s}\left(\bmod p^{\prime} q^{\prime}\right)$

There are three cases.

$\diamond \quad$ If the prover has no knowledge of $x$ in $\Delta_{2}=u^{x}\left(\bmod p^{\prime} q^{\prime}\right)\left(\right.$ or $\Delta_{2}$ has no form of $u^{x}(\bmod$ $\left.p^{\prime} q^{\prime}\right)$ ),

Case 1: If $\Delta_{3} \neq \Delta_{2}, s$ can not be computed under the discrete logarithm assumption.

Case 2: If $\Delta_{3}=\Delta_{2}$, under the S-RSA assumption, the prover has to use the knowledge of double discrete logarithm in $\Delta_{2}$ to compute the $S P K_{A}$ in the step (3), i.e., the knowledge $\left(x_{1}\right.$,

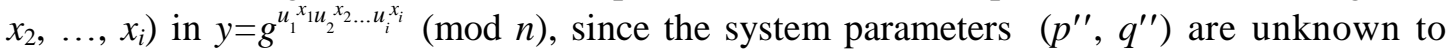
system users;

$\diamond \quad$ If the prover has the knowledge of $x$ in $\Delta_{2}=u^{x}\left(\bmod p^{\prime} q^{\prime}\right)$,

Case 3: According to (\#), $\Delta_{3} \cdot u^{r}=\Delta_{2} \cdot u^{c+s}=u^{x+c+s}\left(\bmod p^{\prime} q^{\prime}\right)$. If $\Delta_{3}$ has no form of $u^{r_{3}}(\bmod$ $p^{\prime} q^{\prime}$ ) when the prover computes it, $s$ can not be computed under the discrete logarithm assumption. Consequently, $\Delta_{3}=u^{r_{3}}\left(\bmod p^{\prime} q^{\prime}\right)$. So $\Delta_{3} \cdot u^{r}=u^{r^{\prime}}=\Delta_{2} \cdot u^{c+s}=u^{x+c+s}\left(\bmod p^{\prime} q^{\prime}\right)$. That is to say, $s=r^{\prime}-x-c$. And because $s=r^{\prime}-x-c$ in Protocol $_{L O G^{2}}$ is in the same form as above $S P K_{\text {LOGnew }}$, the proof of statistical zero-knowledge property can be provided in the same way as the proof of Theorem 4, we omit it here. 
With an off-line TTP, to achieve the proof of knowledge of double discrete logarithm, i.e., the proof of $x$ in $y=g^{u^{x}}(\bmod n)$, the prover does as follows.

Before performing Protocol $L_{L G^{2}}{ }_{2} 2\left(\right.$ or Protocol $_{L O G^{2}}{ }_{3}$ ), the prover has an $A_{\Theta}=g^{u^{r} \Theta}(\bmod n)$ with $r_{\Theta} \in\{0,1\}^{\varepsilon\left(l_{G}+2\right)}$ which had been generated by himself and the construction of which had been verified by the TTP using the previous common method. Then the TTP signed $A_{\Theta}$, e.g., using $\operatorname{SPK}_{A_{\Theta}}=\operatorname{SPK}\left(\alpha: A_{T}=A_{\Theta}{ }^{\alpha} \wedge y_{\mathrm{T}}=h^{\alpha}\right)$ to sign $A_{\Theta}$. Note that if unlinkablity and anonymity are desirable, other signature technique (e.g., [9]) can be used.

$\checkmark \quad$ Note that the above process is performed only once, and then the prover can efficiently provide the proof of knowledge of double discrete logarithm any number of times.

$\underline{\text { Protocol }}_{L O G} \underline{\underline{T}}^{2} \_2$ :

(1) The prover chooses $r_{1} \in{ }_{R}\{0,1\}^{\varepsilon\left(l_{G}+2\right)}$, computes commitment $U_{1}=u^{r_{1}}\left(\bmod p^{\prime} q^{\prime}\right)$, then sends $U_{1}$ and $y$ to the verifier;

(2) The verifier chooses the first challenge information $m \in \in_{R}\{0,1\}^{\varepsilon\left(l_{G}+2\right)}$, and then sends $m$ to the prover;

(3) The prover computes $A=A_{\Theta}^{U_{1}}(\bmod n)$ and sends $A_{\Theta}, \operatorname{SPK} K_{A_{\Theta}}$ and $\operatorname{SPK} K_{U_{1}}=\operatorname{SPK}(\alpha$ : $\left.U_{1}=u^{\alpha}\right)(m)$ to the verifier;

(4) The verifier verifies $S P K_{U_{1}}$ and $S P K_{A_{\Theta}}$, chooses the second challenge information $c \in$ $R\{0,1\}^{k}$ and sends it to the prover;

(5) The prover computes the $s=r_{\Theta}+r_{1}-x-c$ and send s to the verifier;

(6) The verifier verifies $A=y^{u^{c+s}}(\bmod n)$.

Theorem 6. Under the Discrete Logarithm assumption and the S-RSA assumption, the interactive protocol Protocol $L_{\mathrm{OG}^{2}}{ }_{2} 2$ is an honest-verifier statistical zero-knowledge proof of knowledge of the double discrete logarithm of $y=g^{u^{x}}(\bmod n)$.

Proof.

According to the $S P K_{U_{1}}$ and $S P K_{A_{\Theta}}$ in the protocol Protocol $L_{L G^{2}} 2, A=A_{\Theta}^{U_{1}}=g^{u^{r} \ominus \cdot U_{1}}=$ $g^{u^{r} \ominus \cdot u^{r_{1}}}=g^{u^{r_{\Theta} r_{1}}}=g^{u^{r}}(\bmod n)$, where the $r_{\Theta}$ and $r_{1}$ are all known to the prover, i.e., $r$ is known to the prover.

That is to say, we have $A=g^{\alpha}(\bmod n)$ with some known $\alpha$.

From the step (6), $A=y^{u^{c+s}}(\bmod n)$. So $A=g^{u^{r}}=y^{u^{c+s}}(\bmod n) \ldots \ldots \ldots \ldots \ldots \ldots \ldots \ldots \ldots \ldots \ldots \ldots$ (\#1).

In the step (6) of Protocol $_{L O G^{2}}{ }_{2} 2, A=y^{u^{c+s}}(\bmod n)$. So to the prover, $y=A^{\left(u^{c+s}\right)^{-1}}=g^{\alpha \cdot\left(u^{c+s}\right)^{-1}}$ $=g^{A}(\bmod n)$ with some known $\Delta$.

From (\#1), $A=g^{u^{r}}=y^{u^{c+s}}=g^{\Delta \cdot u^{c+s}}(\bmod n)$. That is to say, $u^{r}=\Delta \cdot u^{c+s}\left(\bmod p^{\prime} q^{\prime}\right)$. Note that the $r$ and $\Delta$ are known to the prover before computing $s$. So if $\Delta$ has no form of $u^{x}\left(\bmod p^{\prime} q^{\prime}\right)$, the prover has to compute the discrete logarithm to the base $u$ when computing the response $s$. Therefore, $u^{r}=\Delta \cdot u^{c+s}=u^{x} \cdot u^{c+s}=u^{x+c+s}\left(\bmod p^{\prime} q^{\prime}\right)$. We have $r=x+c+s$, since $\left(p^{\prime \prime}, q^{\prime \prime}\right)$ are secret system parameters. That is to say, $x=r-c-s$. So the Protocol ${ }_{L O G}{ }^{2} \_2$ is a protocol of the knowledge proof of $x$ in $y=g^{u^{x}}(\bmod n)$. And because $s=r-x-c$ is the only one which is not a standard zero-knowledge proof form, we can provide the proof of statistical zero-knowledge property in the same way as the proof of the Theorem 4, we omit it here.

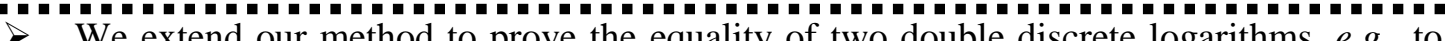
We extend our method to prove the equality of two double discrete logarithms, e.g., to prove that $x_{1}=x_{2}$ in $y_{1}=g^{u^{x_{1}}}(\bmod n)$ and $y_{2}=h^{u^{x_{2}}}(\bmod n)$ in a zero-knowledge way. It can be done as follows.

Protocol $_{L O G}^{2} \underline{3}^{3}$ :

Step (1) and (2) is the same as them in Protocol $_{L_{O G}{ }^{2} \_2 \text {; }}$ 
(3) The prover computes $A=A_{\Theta}{ }^{U_{1}}=g^{u^{r+r_{1}}}=g^{u^{r}}(\bmod n)$ and $B=h^{u^{r}}(\bmod n)$ sends $S P K_{A B U_{1}}=S P K\left(\alpha, \beta, \gamma: A=A_{\Theta}{ }^{{ }^{1}}=g^{\alpha}, B=h^{\alpha}, U_{1}=u^{\gamma}\right)(m), A_{\Theta}$ and $S P K_{A_{\Theta}}$ to the verifier;

(4) The verifier verifies $S P K_{A B U_{1}}$ and $S P K_{A_{\Theta}}$, chooses the challenge information $c \in_{R}\{0,1\}^{k}$ and sends it to the prover;

(5) The prover computes the $s=r_{\Theta}+r_{1}-x-c$ and send s to the verifier;

(6) The verifier verifies $A=y_{1}{ }^{u^{c+s}}(\bmod n)$ and $B=y_{2}{ }^{u^{c+s}}(\bmod n)$.

Theorem 7. Under the Discrete Logarithm assumption and the S-RSA assumption, the interactive protocol Protocol LOG__3 $_{3}$ is an honest-verifier statistical zero-knowledge proof of knowledge of the double discrete logarithm of $y_{1}=g^{u^{x_{1}}}(\bmod n)$ and $y_{2}=h^{u^{x_{2}}}(\bmod n)$ with $x_{1}=x_{2}$.

Proof.

According to the Theorem 6, Protocol $_{L_{O G}{ }^{2} 3} 3$ is an honest-verifier statistical zeroknowledge proof of knowledge of the double discrete logarithm of $y_{1}=g^{u^{x_{1}}}(\bmod n)$ and $x_{1}=r$ $c-s=r_{\Theta}+r_{1}-c-s$.

In the protocol Protocol $_{L O G^{2}}{ }_{3} 3, S P K_{A B U_{1}}=S P K\left(\alpha, \beta, \gamma: A=A_{\Theta}{ }^{{ }^{1}}=g^{\alpha}, B=h^{\alpha}, U_{1}=u^{\gamma}\right)(m)$ proves the exponents of $A$ and $B$ are equal. So under the discrete logarithm assumption, $B=h^{u^{r}}$ (mod $n)$ with $r=r_{\Theta}+r_{1}$. That is to say, $B=y_{2}{ }^{u^{c+s}}=h^{u^{r}}=h^{u^{r} \Theta^{+r_{1}}}(\bmod n)$. For the same reason of the Theorem 6, $x_{2}=r-c-s=r_{\Theta}+r_{1}-c-s$ and $y_{2}=h^{u^{x_{2}}}(\bmod n)$.

Therefore, $y_{1}=g^{u^{x_{1}}}(\bmod n)$ and $y_{2}=h^{u^{x_{2}}}(\bmod n)$ with $x_{1}=x_{2}$. For the same reason of the Theorem 6, we can provide the proof of the statistical zero-knowledge property in the same way as the proof of the Theorem 4, we omit it here.

Base on our Protocol $_{L O G^{2}}$, it easy to provide the noninteractive version of the proofs of knowledge of double discrete logarithm, i.e., the SPK (signature based on a proof of knowledge) of double discrete logarithm

\subsection{New SPK of Double Discrete Logarithm}

$\diamond \quad S P K_{L O G}^{2}{ }_{n e w} 1$

Definition 8 (for Protocol $_{\text {og }^{2}}$ _1). A quadruple (c,s, $\left.s_{1}, s_{2}\right) \in\{0,1\}^{k} \times\{0,1\}^{\varepsilon\left(l_{G}+2\right)} \times$ $\{0,1\}^{\left(2 l_{p}+2\right)} \times\{0,1\}^{\left(2 l_{p}+2\right)}$ satisfying $c=H\left(y^{u^{c+s}}\left\|A_{1}{ }^{c} g^{s_{1}}\right\| A^{c} A_{1}{ }^{s_{1}}\left\|A_{21}{ }^{c} g^{s_{2}}\right\| A_{22}{ }^{c} A_{21}{ }^{s_{2}}\left\|A^{c} A_{22}{ }^{s_{2}}\right\| m\right)$ and $A=y^{u^{c+s}}(\bmod n)$ is an SPK of the double discrete logarithm of a group element $y$ to the base $g$ and $u\left(u_{1 \ldots} u_{i}\right)$, on the message $m \in\{0,1\}^{*}$ and it is denoted as $\boldsymbol{S P K}_{\text {LOG }^{2} \text { new }_{-} 1}\left(\alpha, \alpha_{1}, \ldots, \alpha_{i}: y=g^{u^{\alpha}} \bigvee\right.$

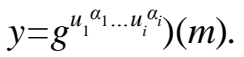

The $S P K_{L O G^{2} \text { new } \_1}\left(\alpha: y=g^{u^{\alpha}}\right)(m)$ can be computed if the double logarithm $x$ of $y$ to the bases $g$ and $u$ (i.e. $x$ in $\left.y=g^{u^{x}}(\bmod n)\right)$ is known, by choosing random $\left(r, r_{1}, r_{2}\right) \in\{0,1\}^{\varepsilon\left(l_{G}+2\right)} \times$ $\{0,1\}^{\varepsilon\left(2 l_{p}+k+2\right)} \times\{0,1\}^{\varepsilon\left(2 l_{p}+2\right)}$ and computing $c, s, s_{1}$ and $s_{2}$ as:

$$
\begin{aligned}
& c=H\left(g^{u^{r}}\left\|g^{r_{1}}\right\| A_{1}^{r_{1}}\left\|g^{r_{2}}\right\| A_{21}{ }^{r_{2}}\left\|A_{22}{ }^{r_{2}}\right\| m\right) \\
& s=r-x-c \\
& s_{1}=r_{1}-c u^{r / 2}\left(\bmod p^{\prime} q^{\prime}\right) \\
& s_{2}=r_{2}-c u^{r / 3}\left(\bmod p^{\prime} q^{\prime}\right)
\end{aligned}
$$

Note that $A=g^{u^{r}}(\bmod n), A_{1}=g^{u^{r / 2}}(\bmod n), A_{21}=g^{u^{r / 3}}(\bmod n), A_{22}=g^{u^{2 r / 3}}(\bmod n) . s_{1}$ in $c=H\left(y^{u^{c+s}}\left\|A_{1}{ }^{c} g^{s_{1}}\right\| A^{c} A_{1}{ }^{s_{1}}\left\|A_{21}{ }^{c} g^{s_{2}}\right\| A_{22}{ }^{c} A_{21}{ }^{s_{2}}\left\|A^{c} A_{22}{ }^{s_{2}}\right\| m\right)$ is the proof of $\operatorname{SPK}\left(\alpha: A=g^{\alpha^{2}}\right)$ and $s_{2}$ is the proof of $\operatorname{SPK}\left(\beta: A=g^{\beta^{3}}\right)$. If the prover chooses to provide the proof of $\operatorname{SPK}\left(\alpha_{1}, \ldots, \alpha_{i}\right.$ : $\left.y=g^{u_{1}{ }_{1} \ldots u_{i} \alpha_{i}}\right)$, it is a knowledge proof with leaking the factor 6 of $\left(\alpha_{1}, \ldots, \alpha_{i}\right)$, while if the prover chooses to provide the proof of $\operatorname{SPK}\left(\alpha: y=g^{u^{a}}\right)$, its interactive version $\left(\right.$ Protocol $\left._{\text {LOG }^{2}}{ }^{1}\right)$ is a statistical zero-knowledge proof. 
$\diamond \quad S P K_{L O G}^{2}{ }_{n e w} 2$

Definition 9 (for Protocol $_{L o G^{2}}$ _2). A triple $\left(c, s, s_{1}\right) \in\{0,1\}^{k} \times\{0,1\}^{\varepsilon\left(l_{G}+2\right)+1} \times$ $\{0,1\}^{\varepsilon\left(\varepsilon\left(l_{G}+2\right)+k\right)}$ satisfying $c=H\left(y^{u^{c+s}}\left\|U_{1}^{c} u^{s}\right\| m\right)$ and $A_{\Theta}{ }^{U_{1}}=y^{u^{c+s}}(\bmod n)$ with $\operatorname{SPK} K_{\Theta}=\operatorname{SPK}(\alpha$ : $\left.A_{T}=A_{\Theta}{ }^{\alpha} \wedge y_{\mathrm{T}}=h^{\alpha}\right)$ is an SPK of the double discrete logarithm of a group element $y$ to the base $g$ and $u$, on the message $m \in\{0,1\}^{*}$ and it is denoted as $\boldsymbol{S P K}_{L_{0 G}{ }^{2}{ }_{n e w} 2}\left(\alpha: y=g^{u^{\alpha}}\right)(m)$.

The $S P K_{L O G^{2}{ }_{n+w} 2}\left(\alpha: y=g^{u^{\alpha}}\right)(m)$ can be computed if the double logarithm $x$ of $y$ to the bases $g$ and $u$ (i.e., $x$ in $\left.y=g^{u^{x}}(\bmod n)\right)$ is known, by choosing random $\left(r_{1}, r_{2}\right) \in\{0,1\}^{\varepsilon\left(l_{G}+2\right)} \times$ $\{0,1\}^{\varepsilon\left(\varepsilon\left(l_{G}+2\right)+k\right)}$ and computing $c, s$ and $s_{2}$ as:

$$
\begin{aligned}
& U_{1}=u^{r_{1}}\left(\bmod p^{\prime} q^{\prime}\right) \\
& A=A_{\Theta}{ }^{U_{1}}=g^{u^{r} \Theta^{\cdot} U_{1}}=g^{u^{r_{\Theta}+r_{1}}}(\bmod n) \\
& c=H\left(A\left\|u^{r_{2}}\right\| m\right) \\
& s=r_{\Theta}+r_{1}-x-c \\
& s_{1}=r_{2}-c r_{1}
\end{aligned}
$$

Note that $s$ in $c=H\left(y^{u^{c+s}}\left\|U_{1}^{c} u^{s_{1}}\right\| m\right)$ is the proof of $x$ in $y=g^{u^{x}}(\bmod n)$, and it is a statistical zero-knowledge proof. $s_{1}$ in $c=H\left(y^{u^{c+s}}\left\|U_{1}{ }^{c} u^{s_{1}}\right\| m\right)$ is the proof of $r_{1}$ in $U_{1}=u^{r_{1}}\left(\bmod p^{\prime} q^{\prime}\right)$. And the TTP's signature, $S P K_{A_{\Theta}}$, guarantees that $A_{\Theta}=g^{u^{r} \Theta}(\bmod n)$.

$$
\diamond \quad S P K_{L O G}^{2}{ }_{n e w} 3
$$

Definition 10 (for Protocol PoG $^{2}$ _3). A quadruple $\left(c, s, s_{1}, s_{2}\right) \in\{0,1\}^{k} \times\{0,1\}^{\varepsilon\left(l_{G}+2\right)+1} \times$ $\{0,1\}^{\varepsilon\left(\varepsilon\left(l_{G}+2\right)+k\right)} \times\{0,1\}^{\left(2 l_{p}+2\right)}$ satisfying $c=H\left(y_{1}{ }^{u^{c+s}}\left\|y_{2}{ }^{u^{c+s}}\right\| U_{1}{ }^{c} u^{s_{1}}\left\|A^{c} g^{s_{2}}\right\| B^{c} h^{s_{2}} \| m\right), A=A_{\Theta}{ }^{U_{1}}=y_{1}{ }^{c^{c+s}}$ $(\bmod n)$ and $B=y_{2}{ }^{u^{c+s}}(\bmod n)$ with $\operatorname{SPK} K_{A_{\Theta}}=\operatorname{SPK}\left(\alpha: A_{T}=A_{\Theta}{ }^{\alpha} \wedge y_{\mathrm{T}}=h^{\alpha}\right)$ is an SPK of the double discrete logarithms of two group elements $y_{1}$ and $y_{2}$, on the message $m \in\{0,1\}^{*}$ and it is denoted as $\boldsymbol{S P K}_{\text {LOG }^{2}{ }_{n e w \_} \mathbf{3}}\left(\alpha: y_{1}=g^{u^{\alpha}} \wedge y_{2}=h^{u^{\alpha}}\right)(m)$.

The $\operatorname{SPK}_{L_{O G}{ }_{n e w} 3}\left(\alpha: y_{1}=g^{u^{\alpha}} \wedge y_{2}=h^{u^{\alpha}}\right)(m)$ can be computed if the double logarithms $x$ of $y_{1}$ and $y_{2}\left(\right.$ i.e. $x$ in $y_{1}=g^{u^{x}}(\bmod n)$ and $\left.y_{2}=h^{u^{x}}(\bmod n)\right)$ is known, by choosing random $\left(r_{1}, r_{2}\right.$, $\left.r_{3}\right) \in\{0,1\}^{\varepsilon\left(l_{G}+2\right)} \times\{0,1\}^{\varepsilon\left(\varepsilon\left(l_{G}+2\right)+k\right)} \times\{0,1\}^{\varepsilon\left(2 l_{p}+2\right)}$ and computing $c, s$ and $s_{2}$ as:

$$
\begin{aligned}
& U_{1}=u^{r_{1}}\left(\bmod p^{\prime} q^{\prime}\right) \\
& A=A_{\Theta}^{U_{1}}=g^{u^{r} U_{1}}=g^{u^{r_{\Theta}+r_{1}}}(\bmod n) \\
& B=h^{u^{r+r_{1}}}(\bmod n) \\
& c=H\left(A\|B\| u^{r_{2}}\left\|g^{r_{3}}\right\| h^{r_{3}} \| m\right) \\
& s=r_{\Theta}+r_{1}-x-c \\
& s_{1}=r_{2}-c r_{1} \\
& s_{2}=r_{3}-c u^{r_{\Theta}+r_{1}}\left(\bmod p^{\prime} q^{\prime}\right)
\end{aligned}
$$

Note that $s_{2}$ in $c=H\left(y_{1}{ }^{u^{c+s}}\left\|y_{2}{ }^{u^{c+s}}\right\| U_{1}{ }^{c} u^{s_{1}}\left\|A^{c} g^{s_{2}}\right\| B^{c} h^{s_{2}} \| m\right)$ is $\operatorname{SPK}\left(\alpha: A=g^{\alpha}, B=h^{\alpha}\right)$.

\section{Efficiency Analysis}

Our $S P K_{L O G^{2}}{ }^{n}$ ew improves the time complexity and the space complexity from $O(k)$ of the existing schemes $[10,13,19,27,30-32,35,39]$ to $O(1)$.

Following the scheme [10], we take $\varepsilon=4 / 3$ and $\lambda=170$ when counting the storage space. In the system, we take the public modulus $n(=p q)$ to be 1024 bits and $c$ (the output of hush function) to be 160 bits. Note that the cost of our storage space can be significantly reduced if using other groups (e.g., elliptic curve groups).

For achieving a clear comparison of computation costs, we consider the exponentiation costs which are the main computation costs. In a good implementation $[18,48]$, the computation of the multi-based exponentiation does not take far more time 
than a single exponentiation. Normally, a multi-based exponentiation takes only $10 \%$ more time compared with a single-based exponentiation (assume that one multi-EXP operation multiplies up to 3 exponentiations). So for comparing clearly, we treat single exponentiation as multi-based exponentiation uniformly.

The existing signature schemes (SPK) based on proofs of knowledge of the double discrete logarithm [10, 13, 19, 27, 30-32, 35, 39] take $4 k$ modular exponentiations to generate the signature and verify it, and in their systems, the probability of forging the signature $\operatorname{Prob}_{\text {forging }}=2^{-k}$. And our scheme takes $O(1)$ modular exponentiations, while in our system, the probability of forging the signature Prob $_{\text {forging }}<2^{-k}$. As mentioned before, usually, $k>80$ in applications, so for achieving the same security level, our scheme significantly improves the efficiency.

According to the table 1, with an off-line TTP, our $S P K_{L_{O G}{ }^{2}{ }_{n+w} 3}$ has provided an efficient solution to the open problem of proving the equality of two double discrete logarithms, e.g., proving that $x_{1}=x_{2}$ in $y_{1}=g^{u^{x_{1}}}(\bmod n)$ and $y_{2}=h^{u^{x_{2}}}(\bmod n)$ in a zeroknowledge way.

\section{Conclusion}

We provide the first zero-knowledge proof of double discrete logarithm with constant complexity, and we also provide the first zero-knowledge proof of equality of double discrete logarithms. We redesign the basic construction of knowledge proof, so our knowledge proofs of double discrete logarithm are much more efficient than the existing schemes. The time complexity and the space complexity all change in quality. The zero-knowledge proof of equality of double discrete logarithms (i.e., our $S P K_{L_{O G}{ }^{2} \text { new }_{3} 3}$ ) is efficiently realized for the first time. Though being different from $S P K_{L G^{2}{ }_{n e w}-1}$, there is an off-line TTP, the prover can perform zero-knowledge proofs with any verifier any number of times after getting TTP's signature on his parameter just once. So for solving the open problem of zero-knowledge proving equality of double discrete logarithms, our idea is feasible in applications. Our contributions will make the systems $[10,13,19,27,30-32,35,39]$ which use zero-knowledge proofs of double discrete logarithm more efficient and practical.

\section{Table 1. The Comparison between $S P K_{L O G}{ }^{2}$ new and Existing $S P K_{L O G}{ }^{2}$ Schemes}

\begin{tabular}{|c|c|c|c|c|c|c|c|c|c|c|}
\hline \multirow{3}{*}{\multicolumn{2}{|c|}{\begin{tabular}{|c} 
\\
Existing $S P K_{\text {LOG }^{2}}$ \\
schemes \\
in $[10,13,19,27$ \\
$30,31,32,35,39]$ \\
\end{tabular}}} & \multirow{3}{*}{$\begin{array}{c}\mathbf{I} \\
O(k)\end{array}$} & \multirow{3}{*}{$\begin{array}{r}\mathbf{I}_{(1)} \\
\\
4 k\end{array}$} & \multicolumn{2}{|c|}{$\Pi_{(2)}$} & \multirow{3}{*}{$\begin{array}{c}\text { III } \\
O(k)\end{array}$} & \multirow{3}{*}{$\begin{array}{c}\text { IV } \\
\begin{array}{c}(160+227 k) \\
\text { bits }\end{array}\end{array}$} & \multirow{3}{*}{$\begin{array}{c}\mathrm{V} \\
=2-k\end{array}$} & \multirow{3}{*}{$\begin{array}{r}\text { VI } \\
k>80\end{array}$} & \multirow{3}{*}{$\begin{array}{c}\text { VII } \\
S P K\left(\alpha: y=g_{u}^{u^{g}}\right)(m)\end{array}$} \\
\hline & & & & $P$ & $2 k$ & & & & & \\
\hline & & & & V & $2 k$ & & & & & \\
\hline \multirow{5}{*}{$\begin{array}{c}\text { Our } \\
\text { schemes }\end{array}$} & \multirow{2}{*}{$S P K_{L O G^{2}{ }^{2} w_{-} 1}$} & \multirow{2}{*}{$O(1)$} & \multirow{2}{*}{18} & $P$ & 11 & \multirow{2}{*}{$O(1)$} & \multirow{2}{*}{5507 bits * } & \multirow{2}{*}{$<2^{-k}$} & \multirow{2}{*}{$k>80$} & \multirow{2}{*}{$\begin{array}{c}\operatorname{SPK}\left(\alpha, \alpha_{1}, \ldots, \alpha_{i}: y=g_{u}^{u}\right. \\
\left.\vee y=g_{w}^{u_{1}}, \ldots u_{i}^{a_{i}}\right)(m)\end{array}$} \\
\hline & & & & $V$ & 7 & & & & & \\
\hline & \multirow{2}{*}{$S P K_{L O G^{2}{ }^{2} w_{-} 2}$} & \multirow{2}{*}{$O(1)$} & \multirow{2}{*}{9} & $P$ & 3 & \multirow{2}{*}{$O(1)$} & \multirow{2}{*}{4073 bits * } & \multirow{2}{*}{$<2^{-k}$} & \multirow{2}{*}{$k>80$} & \multirow{2}{*}{$\operatorname{SPK}\left(\alpha: y=g_{m}^{u^{a}}\right)(m)$} \\
\hline & & & & $V$ & 6 & & & & & \\
\hline & $S P K_{L O G^{2}}{ }^{2} w_{-} 3$ & $O(1)$ & 16 & $P$ & 7 & $O(1)$ & 5097 bits * & $<2^{-k}$ & $k>80$ & $S P K\left(\alpha: y_{1}=g_{u^{u}}^{a}\right.$ \\
\hline
\end{tabular}




$\begin{array}{ll}P: & \text { the prover } \\ V: & \text { the verifier } \\ \mathrm{I}: & \text { the time complexity } \\ \mathrm{II}_{(1)}: & \text { the total computation of multi-based exponentiations } \\ \mathrm{II}_{(2)}: & \text { the multi-based exponentiations from each participant } \\ \mathrm{III}: & \text { the space complexity } \\ \mathrm{IV}: & \text { the storage space of the signature } \\ \mathrm{V}: & \text { the probability of forging the signature } \\ \mathrm{VI}: & \text { the requirement of security parameter } \\ \mathrm{VII}: & \text { the function of each scheme } \\ *: & \text { the storage costs in RSA-groups }\end{array}$

\section{Acknowledgements}

This work was supported by The National Natural Science Foundation of China (No: 61271220), Security Management of Information Content Innovation Base (No: TS0010303001), National Engineering Laboratory for Information Content Analysis Technology (No: GT036001), and Natural Science Foundation of Ningbo (No: 2012A610064).

\section{References}

[1] L. Babai, "Trading group theory for randomness", In Proceedings of the Seventeenth Annual ACM Symposium on Theory of Computing, (1985), pp. 421-429.

[2] L. Babai and S. Moran, "Arthur - Merlin games: A randomized proof system, and a hierarchy of complexity classes", Journal of Computer and System Sciences, vol. 36, (1988).

[3] S. Goldwasser, S. Micali and C. Rackoff, "The knowledge complexity of interactive proof systems", In Proc. 27th Annual Symposium on Foundations of Computer Science, (1985), pp. 291-304.

[4] O. Goldreich, S. Micali and A. Wigderson, "Proofs that Yield Nothing but Their Validity and a Methodology of Cryptographic Protocol Design”, Proceedings of FOCS, (1986), pp. 174-187.

[5] O. Goldreich, S. Micali and A. Wigderson, "How to prove all NP statements in zero-knowledge and a methodology of cryptographic protocol design”, In Andrew M. Odlyzko, editor, Advances in Cryptology CRYPTO '86, Lecture Notes in Computer Science, vol. 263, (1987), pp. 171-185.

[6] G. Brassard, D. Chaum and C. Crépeau, "Minimum disclosure proofs of knowledge", Journal of Computer and System Sciences, (1988), pp. 156-189.

[7] G. Brassard and C. Crépeau, "Non-transitive transfer of confidence: a perfect zero-knowledge interactive protocol for SAT and beyond", In Proc. 27th IEEE Symp. Found. Comp. Sc., (1986), pp. 188-195.

[8] D. Chaum, "Demonstrating that a public predicate can be satisfied without revealing any information about how", In Andrew M. Odlyzko, editor, Advances in Cryptology - CRYPTO '86, Lecture Notes in Computer Science, vol. 263, (1987), pp. 195-199.

[9] G. Ateniese, J. Camenisch, M. Joye and G. Tsudik, "A practical and provably secure coalition-resistant group signature scheme", Advances in Cryptology CRYPTO, (2000), pp. 255-270.

[10] J. Camenisch, "Group signature schemes and payment systems based on the discrete logarithm problem", $\mathrm{PhD}$ thesis, vol. 2 of ETH Series in Information Security an Cryptography, Hartung-Gorre Verlag, Konstanz, ISBN 3-89649-286-1, (1998).

[11] J. Camenisch and M. Michels, "A group signature scheme based on an RSA-variant", Technical Report RS98-27, BRICS, University of Aarhus, (1998) November.

[12] J. Camenisch and M. Michels, "Proving in zero-knowledge that a number is the product of two safe primes", Advances in Cryptology EUROCRYPT'99, (1999), pp. 107-122.

[13] J. Camenisch and M. Stadler, "Efficient group signature schemes for large groups", Advances in Cryptology CRYPTO'97, (1997), pp. 410-424.

[14] J. Camenisch, S. Hohenberger, and A. Lysyanskaya, "Compact e-cash", Advances in Cryptology EUROCRYPT, (2005), pp. 302-321.

[15] M. Au, Q. Wu, W. Susilo and Y. Mu, "Compact E-Cash from Bounded Accumulator" In Proceedings of CTRSA'07 LNCS, vol. 4377, (2007), pp. 178-195.

[16] M. Au, W. Susilo and Y. Mu, "Practical compact e-cash", Proceedings of the 12th Australasian conference on Information security and privacy, (2007), pp. 431-445. 
[17] M. Au, W. Susilo and Y. Mu, "Practical anonymous divisible e-cash from bounded accumulators", Financial Cryptography and Data Security, (2008), pp. 287-301.

[18] M. Au, W. Susilo and Y. Mu, "Proof-of-Knowledge of Representation of Committed Value and Its Applications", Information Security and Privacy, (2010), pp. 352-369.

[19] S. Canard and A. Gouget, "Divisible e-cash systems can be truly anonymous", Advances in CryptologyEUROCRYPT, (2007), pp. 482-497.

[20] S. Canard, C. Delerablée, A. Gouget, E. Hufschmitt, F. Laguillaumie, H. Sibert, J. Traoré and D. Vergnaud, "Fair E-Cash: Be Compact, Spend Faster", Information Security, (2009), pp. 294-309.

[21] S. Canard and J. Traoré, "On fair e-cash systems based on group signature schemes", ACISP, (2003), pp. 237-248.

[22] A. Lysyanskaya and Z. Ramzan, "Group blind digital signatures: A scalable solution to electronic cash", FC'98, (1998), pp. 197-238.

[23] G. Maitland and C. Boyd, "Fair electronic cash based on a group signature scheme", Information and Communications Security, (2001), pp. 461-465.

[24] X. Zhao and F. Zhang, "Times Limited Accountable Anonymous Online Submission Control System from Single-Verifier k-times Group Signature”, Informatica, vol. 36, (2012), pp. 75-82.

[25] M. H. Au, W. Susilo, Y. Mu and S. S. M. Chow, "Constant-Size Dynamic k-Times Anonymous Authentication”, IEEE SYSTEMS JOURNAL, SN 1932-8184, vol. 7, no. 2, (2013), pp. 249-261.

[26] B. Lian, G. Chen and J. Li, "Provably secure E-cash system with practical and efficient complete tracing", International Journal of Information Security, DOI 10.1007/s10207-014-0240-2, vol. 13, issue 3, (2014), pp. 271-289.

[27] M. H. Au, "Contribution to Privacy-Preserving Cryptographic Techniques", Ph.D. Thesis, University of Wollongong, (2009) May.

[28] J. Camenisch, R. Chaabouni and A. Shelat, "Efficient protocols for set membership and range proofs", Advances in Cryptology-ASIACRYPT 2008, (2008), pp. 234-252.

[29] J. Camenisch, A. Kiayias and M. Yung, "On the portability of generalized schnorr proofs", Advances in Cryptology-EUROCRYPT, (2009), pp. 425-442.

[30] G. Ateniese and G. Tsudik, "Some open issues and new directions in group signatures", In: Franklin, M. (ed.) FCT LNCS, vol. 1684, (1999), pp. 196-211.

[31] G. Ateniese, D. Song and G. Tsudik, "Quasi efficient revocation group signatures", In: Blaze, M. (ed.) FC 2002 LNCS, vol. 2357, (2003), pp. 183-197.

[32] L. Bussard, Y. Roudier and R. Molva, "Untraceable secret credentials: trust establishment with privacy", Pervasive Computing and Communications Workshops, In: Proceedings of the Second IEEE Annual Conference, (2004) March 14-17, pp. 122-126.

[33] H. M. Elkamchouchi, M. E. Nasr and R. Esmail, "New public key techniques based on double discrete logarithm problem", Radio Science Conference, NRSC 2004, In: Proceedings of the Twenty-First National, vol. C23, (2004), pp. 1-9.

[34] M. A. Kula, “A cryptosystem based on double exponentiation”, Tatra Mt. Math. Publ., vol. 25, (2002), pp. 67-80.

[35] A. Lysyanskaya and Z. Ramzan, "Group blind digital signatures: A scalable solution to electronic cash", In: Hirschfeld, R. (ed.) FC LNCS, vol. 1465, (1998), pp. 184-197.

[36] M. Stadler, "Publicly verifiable secret sharing. In: Maurer, U.M. (ed.) EUROCRYPT 1996 LNCS, vol. 1070, (1996), pp. 190-199.

[37] R. Tso, T. Okamoto and E. Okamoto, "Practical strong designated verifier signature schemes based on double discrete logarithms. In: Feng, D., Lin, D., Yung, M. (eds.) CISC 2005 LNCS, vol. 3822, (2005), pp. 113-127.

[38] G. Wang and S. Qing, "Security flaws in several group signatures proposed by Popescu", In: Gervasi, O., Gavrilova, M.L., Kumar, V., Lagan' a, A., Lee, H.P., Mun, Y., Taniar, D., Tan, C. J. K. (eds.) ICCSA 2005 LNCS, vol. 3482, (2005), pp. 711-718.

[39] A. Miyaji and K. Umeda, "a fully-functional group signature scheme over only known-order group", Applied Cryptography and Network Security, Lecture Notes in Computer Science, vol. 3089, (2004), pp. 164-179.

[40] C. Konoma, M. Mambo and H. Shizuya, "The computational difficulty of solving cryptographic primitive problems related to the discrete logarithm problem", IEICE Transactions on Fundamentals of Electronics, Communications and Computer Sciences E88-A(1), (2005), pp. 81- 88.

[41] G. C. Meletiou and A. Winterhof, "Interpolation of the Double Discrete Logarithm", J. von zur Gathen, J.L. Imãna, and C, .K. Ko,c (Eds.), WAIFI 2008, LNCS 5130, (2008), pp. 1-10.

[42] A. Fiat and A. Shamir, "How to prove yourself: Practical solution to identification and signature problems", In Andrew M. Odlyzko, editor, Advances in Cryptology - CRYPTO '86, Lecture Notes in Computer Science, vol. 263, (1987), pp. 186-194.

[43] E. Fujisaki and T. Okamoto, "Statistical zero knowledge protocols to prove modular polynomial relations", Advances in Cryptology_CRYPTO'97, (1997), pp. 16-30. 
[44] N. Bari and B. Pfitzmann, "Collision-free accumulators and fail-stop signature schemes without trees", In Advances in Cryptology - EUROCRYPT’ 97, LNCS, vol. 1233, (1997), pp. 480-494.

[45] S. Brands and C. V. W. E. Informatica, "An efficient off-line electronic cash system based on the representation problem" Citeseer, (1993).

[46] C. P. Schnorr, "Efficient signature generation for smart cards", Journal of Cryptology, (1991), pp. $239-252$.

[47] M. Bellare and P. Rogaway, "Random oracles are practical: A paradigm for designing efficient protocols", Proceedings of the 1st ACM conference on Computer and communications security, (1993), pp. 62-73.

[48] J. Camenisch and J. Groth, "Group signatures: Better efficiency and new theoretical aspects", Security in Communication Networks, (2005), pp. 120-133.

\section{Authors}

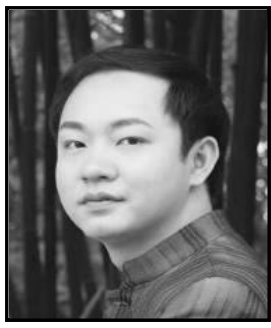

Bin Lian, He received his M.S. degree in cryptography from Southwest Jiaotong University in 2005, Chengdu. Now he works at Ningbo Institute of Technology, Zhejiang University. Currently, he is a Ph.D. candidate in the School of Information Security Engineering, Shanghai Jiao Tong University, Shanghai. His research interests lie in cryptography and technology of network security.

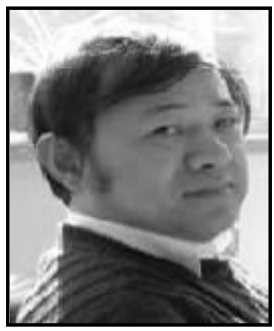

Gongliang Chen, He received his B.S. in Peking University, and M.S. degree in Chinese Academy of Science. In 1993, he received his Ph.D. degree in Université de Saint Etienne, France. He is also a visiting scholar of Université Paris VI, France. He is currently a professor at the School of Information Security Engineering, Shanghai Jiao Tong University, Shanghai. His main research area includes cryptographic theory, technology of network security.

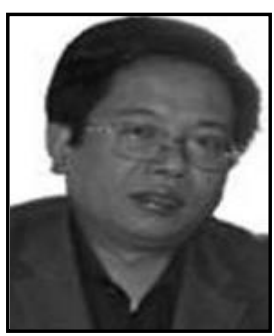

Jianhua Li, He received his Ph.D. degree in Shanghai Jiao Tong University, Shanghai in 1998. He is now a professor and doctoral supervisor at the School of Information Security Engineering, Shanghai Jiao Tong University, Shanghai. He is also the director of National Engineering Laboratory for Information Content Analysis Technology. His recent research directions are cryptography and technology of network security. 